\title{
1 An affinity-matured DLL4 ligand for broad-spectrum activation and 2 inhibition of Notch signaling
}

4 David Gonzalez-Perez ${ }^{1}$, Satyajit Das ${ }^{2}$, Elliot Medina ${ }^{1}$, Daniel Antfolk ${ }^{1}$, Emily D. Egan ${ }^{3}$, Stephen 5 C. Blacklow ${ }^{3}$, Paulo C. Rodriguez ${ }^{2}$, and Vincent C. Luca ${ }^{*}$.

${ }^{1}$ Department of Drug Discovery, Moffitt Cancer Center, Tampa, FL 33602, USA

$8 \quad 2$ Department of Immunology, Moffitt Cancer Center, Tampa, FL 33612, USA

${ }^{3}$ Department of Biological Chemistry and Molecular Pharmacology, Harvard Medical School,

10 Boston, Massachusetts 02115; Department of Cancer Biology, Dana Farber Cancer Institute,

11 Boston, Massachusetts 02115, USA

$12{ }^{*}$ Corresponding author: vince.luca@moffitt.org

14 Key words: Notch signaling, receptor biology, protein engineering, $\mathrm{T}$ cell activation

\section{ABSTRACT}

17 The Notch pathway regulates cell fate decisions and is an emerging target for regenerative and 18 cancer therapies. Recombinant Notch ligands are attractive candidates for modulating Notch 19 signaling; however, their intrinsically low receptor-binding affinity restricts their utility in

20 biomedical applications. To overcome this limitation, we evolved variants of the ligand Delta-like 214 (DLL4) with enhanced affinity and cross-reactivity. A consensus variant with maximized

22 binding affinity, Delta ${ }^{\mathrm{MAX}}$, engages human and murine Notch receptors with 500- to 1000-fold 23 increased affinity compared to wild-type human DLL4. Delta ${ }^{\mathrm{MAX}}$ also potently activates human 24 Notch in plate-bound, bead-bound, and cellular formats. When administered as a soluble decoy, 25 Delta ${ }^{\mathrm{MAX}}$ inhibits Notch activation in response to either Delta-like (DLL) or Jagged (Jag) ligands, 26 highlighting its utility as both an agonist and antagonist. Finally, we demonstrate that Delta ${ }^{\mathrm{MAX}}$ 27 stimulates increased proliferation and expression of effector mediators in primary activated human 28 T cells. Taken together, our data defines Delta ${ }^{\mathrm{MAX}}$ as a versatile biotechnological tool for broad29 spectrum activation or inhibition of Notch signaling. 


\section{INTRODUCTION}

34 The Notch pathway is a conserved signaling system that regulates metazoan development and cell

35 fate decisions. In mammals, the core Notch signaling network consists of four Notch receptor 36 paralogs (Notch1-4) and the activating ligands Delta-like 1 (DLL1), Delta-like 4 (DLL4), Jagged1

37 (Jag1), and Jagged2 (Jag2). Notch signaling occurs when cells expressing Notch proteins (signal 38 receivers) interact with adjacent cells expressing DLL or Jag ligands (signal senders) ${ }^{1-4}$. Following 39 ligand engagement, endocytosis of the DLL or Jag protein into the sender cell exerts a "pulling" 40 force that destabilizes the negative regulatory region (NRR) of the Notch extracellular domain $41(\mathrm{ECD})^{5,6}$. This mechanical tension exposes a proteolytic cleavage site (S2) that is processed by the 42 ADAM10 metalloprotease ${ }^{7,8}$. ADAM10 cleavage results in the shedding of the Notch ECD, which 43 in turn sensitizes the Notch transmembrane domain to cleavage by the intramembrane protease 44 gamma-secretase ${ }^{1,9,10}$. This second proteolytic event releases the Notch intracellular domain 45 (NICD) from the plasma membrane and it translocates to the nucleus to serve as a transcriptional 46 cofactor ${ }^{1,2,11}$.

Notch extracellular domains (ECDs) are comprised of 29 to 36 epidermal growth factor (EGF) domains and the juxtamembrane negative regulatory region (NRR). Ligand ECDs also have a modular structure and contain an N-terminal C2-like (MNNL) domain, a Delta/Serrate/Lag3 (DSL) domain, 6 to 16 EGF domains, and a cysteine rich domain that is only present in Jag proteins. Through structural, biochemical, and cell-based studies, we and others have determined that the C2, DSL, and EGF1-3 region of DLL or Jag engage five centrally positioned Notch EGF domains (EGF8-12 in Notch1) to initiate signaling ${ }^{4,12-15}$. Interactions between Notch and ligand ECDs are low affinity $4,13,16$, and co-crystallization of rat DLL4:Notch1 and Jag1:Notch1 complexes required the incorporation of affinity-enhancing mutations into the ligands ${ }^{14,15}$. Despite the low affinity of receptor for ligand, Notch signaling occurs productively in vivo, because 58 receptor-ligand interactions may be strengthened by catch bond formation or multivalent 59 binding ${ }^{15,17-19}$.

Although the Notch transcriptional machinery is broadly conserved, preferential interactions have been described between receptor and ligand subtypes ${ }^{1}$. For example, DLL4 is a higher affinity 
63 ligand for Notch1 than is DLL1 ${ }^{13}$, while DLL1 activates Notch1 and Notch2 roughly 64 equivalently ${ }^{16}$. Signaling through specific Notch-ligand pairs is also associated with distinct 65 functional outcomes. During angiogenesis, Notch1:DLL4 interactions inhibit tip sprouting and 66 Notch1:Jag1 interactions promote vessel growth ${ }^{20}$, and Jagged2:Notch3 interactions stimulate the 67 differentiation of $\gamma / \delta \mathrm{T}$ cells ${ }^{21}$. Comparative studies have begun to identify differences in the 68 structures ${ }^{15}$, binding affinities ${ }^{13}$, and signaling dynamics ${ }^{19}$ of selected ligands that may contribute 69 to their unique functional properties. However, the precise molecular mechanisms controlling 70 receptor- and ligand-specific signaling remain unclear. Depending on cellular context, Notch signaling may induce the differentiation or proliferation of stem cells, and the broad influence of Notch on stem cell behavior has made the pathway an

74 attractive target for regenerative therapeutics. Moreover, Notch signaling has been shown to have context-dependent tumor suppressor or oncogenic functions in human cancers ${ }^{22}$. The pleiotropic effects of Notch signaling suggest that both agonists and antagonists of Notch signaling will be valuable for biomedical applications. Several antagonists are currently in clinical trials for the treatment of cancer, including gamma-secretase inhibitors and monoclonal antibodies targeting individual Notch ECDs ${ }^{23-25}$. On the other hand, the development of Notch agonists has been challenging because soluble drugs cannot exert the mechanical force necessary for receptor activation. Thus far, a single agonist antibody has been described for Notch3, which may be uniquely susceptible to antibody-mediated activation due to the inherent instability of the Notch3

$83 \mathrm{NRR}^{26,27}$.

Recombinant DLL or Jag ECDs are attractive "one-size-fits-all” candidates for activating or inhibiting Notch signaling. In their soluble form, ligand ECDs act as untethered decoys that bind to Notch receptors and inhibit ligand-mediated activation. The on-target specificity of ligand ECDs may also be advantageous given that gamma-secretase cleaves a myriad of cellular substrates that are unrelated to the Notch pathway ${ }^{28}$. Alternatively, surface- or matrix-tethered DLL or Jag ligands stimulate Notch signaling, presumably because immobilization provides sufficient resistance force

91 for receptor activation. Despite their potential to function as universal modulators of the Notch 92 pathway, the intrinsically low affinities of DLL and Jag ligands limits their practical utility as 
93 biomedical tools. Additionally, the preferential binding observed between certain receptor:ligand

94 pairs may restrict the activity of a given ligand to a subset of Notch paralogs.

To overcome the biochemical limitations of endogenous Notch ligands, we used yeast display and mutations from multiple directed evolution campaigns, we developed a consensus variant, named Delta $^{\mathrm{MAX}}$, that binds with greatly enhanced affinity to human and murine Notch receptor paralogs. We determined that Delta ${ }^{\text {MAX }}$ is a more potent Notch activator than WT DLL4 in several commonly used ligand-presentation formats, and that the proliferation and expression of effector mediators in primary $\mathrm{T}$ cells is enhanced following conditioning stimulation with Delta ${ }^{\mathrm{MAX}}$. Furthermore, the soluble Delta ${ }^{\mathrm{MAX}}$ ECD functions as a potent, "pan-Notch" inhibitor by competing for the Notch ligand-binding site. Our collective insights demonstrate that affinity-matured Notch

\section{RESULTS}

108 Structure-guided engineering of high-affinity DLL4 variants. We employed a structure-guided 109 engineering strategy to evolve broadly-reactive, high-affinity DLL4 variants. Crystal structures of 110 DLL4:Notch1 and Jag1:Notch1 complexes revealed that the ligand C2 and DSL domains engage 111 the Notch1 EGF12 and EGF11 domains, respectively. In each of these structures, the C2:EGF12 112 interface was named Site 1 and the DSL:EGF11 interface was named Site 2. However, an 113 additional "Site 3" binding interface was also visualized in the Jag1:Notch1 structure and forms 114 between Jag1 EGF1-3 and Notch1 EGF8-10 ${ }^{14,15}$. Solution binding studies demonstrated that Site 1153 contributes substantially to Jag1:Notch1 interactions, but has a minimal effect on DLL4:Notch1 116 interactions ${ }^{15}$. Therefore, we designed a site-directed mutant library to select for DLL4 mutations 117 that recapitulate the Site 3 interaction observed in Notch1:Jag1. The library was generated by 118 varying nine DLL4 residues (H256, N257, T271, L279, F280, T289, S301, N302, Q305) that were 119 analogous to Site 3 interface residues in Jag1 EGF2 and EGF3. We employed a "light mutagenesis" 120 approach in which each interface residue was allowed to encode for the WT DLL4 residue, the 121 equivalent Jag1 residue, or biochemically similar residues (Fig. 1a, 1b). 
123 We used yeast display to select high-affinity Notch binders from our DLL4 mutant library (Fig.

124 1b). The library was stained with Notch1 or Notch3 ECD constructs containing the ligand-binding 125 domains (Notch1 EGF8-12, Notch3 EGF5-12) and several rounds of selections were performed to 126 isolate high-affinity binders. The Notch3-selected yeast had the greatest enrichment of Notch127 binders, and sequencing of individual colonies revealed twenty-one different mutations at interface 128 positions (Fig. 1c, Extended Data Fig. 1). A clone containing five mutations (N257P, T271L, 129 F280Y, S301R, and Q305P) was overrepresented in the samples and was selected for further 130 characterization. This clone is henceforth referred to as DLL4.v2. Analysis of the DLL4.v2 131 sequence revealed that F280Y and S301R are novel mutations that are not present in other 132 mammalian Notch ligands (Extended Data Fig. 2), and that N257P, T271L, and Q305P had 133 converted to the Jag1 residues. The other four mutated positions (DLL4 residues H256, L279, 134 T289, and N302) reverted to the wild-type sequence (Fig. 1c). Notably, N257P was previously 135 introduced into DLL4 to recreate the "DOS motif" found in Jag1 $1^{2,29}$, and this substitution increased 136 receptor binding and signaling ${ }^{30}$.

138 We analyzed the DLL4.v2 mutations in the context of Site 3 of the Notch1:Jag1 complex structure 139 to gain insight into their mechanisms of affinity-enhancement (Fig. 1d, Extended Data Fig. 3). 140 Based on this analysis, we predict that the N257P, T271L, and Q305P mutations enhance binding 141 by improving hydrophobic packing at the binding interface (Extended Data Fig. 3a, 3b, 3c). The 142 F280Y mutation may stabilize the overall fold of the DLL4 protein by replacing the exposed 143 hydrophobic Phe ${ }^{280}$ phenyl group with a more hydrophilic tyrosyl group (Extended Data Fig. 3d). 144 Lastly, we predict that S301R enhances binding by introducing contacts between the guanidinium 145 group of DLL4 $\mathrm{Arg}^{301}$ and the main-chain carbonyl of the Notch1 Cys ${ }^{321}$ and/or the aliphatic side 146 chain of $\mathrm{Val}^{322}$ (Extended Data Fig. 3e).

148 Generation of a high-affinity DLL4 consensus variant. We combined multiple sets of affinity149 enhancing mutations in an attempt to engineer a DLL4 protein with maximal receptor-binding 150 affinity. We used the high-affinity DLL4.v2 variant as a starting point for our construct design. 151 We then engrafted five affinity-enhancing mutations (G28S, F107L, N118I, H194Y, and L206P) 152 from our previously reported rat DLL4 variant (E12) ${ }^{14}$ onto the human DLL4.v2 scaffold (Fig. 153 2a). As the E12 mutations are located within the C2 and DSL domains (Site 1 and Site 2) of DLL4, 
154 we hypothesized that they would have an additive or synergistic effect when combined with the 155 Site 3 mutations of DLL4.v2. The resulting consensus variant, Delta ${ }^{\mathrm{MAX}}$, contains 10 total mutations: G28S, F107L, N118I, H194Y, L206P, N257P, T271L, F280Y, S301R, and Q305P (Fig.

$1572 \mathrm{a})$.

DLL4 variants bind to human and mouse Notch receptors with high-affinity. We used surface plasmon resonance (SPR) to determine the binding affinity between Delta ${ }^{\mathrm{MAX}}$ and the ligandbinding regions of Notch1-4. As a basis for comparison, we also generated a WT DLL4 construct, a DLL4.v2 construct, and a "DLL4.v1" construct containing the five E12 mutations (G28S, F107L, N118I, H194Y, and L206P) (Fig. 2a, Extended Data Fig. 4). Analysis of the SPR data revealed that DLL4.v1, DLL4.v2, and Delta ${ }^{\mathrm{MAX}}$ bound to all four human Notch receptors with enhanced affinity compared to WT DLL4 (Fig. 2b). DLL4.v1 bound to Notch1-4 with 70 to 200-fold increased affinity depending on the receptor subtype, and DLL4.v2 bound to Notch1-4 with 6 to 28-fold higher affinity. The larger contribution of Site 1 and Site 2 mutations from E12 to the interaction is consistent with previous data indicating that the $\mathrm{C} 2$ and DSL domains form the dominant receptor-binding interface. Delta ${ }^{\mathrm{MAX}}$ bound to Notch1 with a dissociation constant $\left(\mathrm{K}_{\mathrm{D}}\right)$ of $24 \mathrm{nM}$, Notch2 with a $\mathrm{K}_{\mathrm{D}}$ of $54 \mathrm{nM}$, Notch3 with a $\mathrm{K}_{\mathrm{D}}$ of $49 \mathrm{nM}$, and Notch4 with a $\mathrm{K}_{\mathrm{D}}$ of 24 nM. Compared to WT DLL4, the nanomolar affinity interactions between Delta ${ }^{\mathrm{MAX}}$ and Notch14 represent enhancements of 1,100-fold, 670-fold, 591-fold, and 532-fold, respectively.

174 In addition to human Notch1-4, we measured the binding affinity between each DLL4 variant and the EGF6-13 region of murine Notch1 (mNotch1 EGF6-13) to test for cross-species reactivity (Fig. 2b, Extended Data Fig. 4, 5). Each ligand bound to murine Notch1 and human Notch1 with comparable affinities, and Delta ${ }^{\mathrm{MAX}}$ bound to mNotch1 EGF6-13 with a $\mathrm{K}_{\mathrm{D}}$ of $24 \mathrm{nM}$, which is nearly identical to its affinity for human Notch1 $(24 \mathrm{nM} \mathrm{K})$. We attempted to fit the SPR data to compare the kinetic binding parameters between Notch and each of the four DLL4 constructs. The

180 rapid dissociation of WT DLL4 precluded kinetic fitting of the binding data, however, visual 181 inspection of the SPR sensograms indicates that the increasing affinities of DLL4.v1, DLL4.v2, 182 and Delta ${ }^{\mathrm{MAX}}$ are linked to progressive decreases in off-rate (Fig. 2c). 
184 Delta ${ }^{\text {MAX }}$ has improved expression and thermostability. We also tested whether the affinity185 enhancing mutations impacted the expression and stability of the Delta ${ }^{\mathrm{MAX}}$ protein. We performed 186 a thermal denaturation experiment using differential scanning fluorimetry (DSF) to compare the 187 melting temperatures of WT DLL4 and Delta ${ }^{\mathrm{MAX}}$. We determined that the melting temperature $188\left(\mathrm{~T}_{\mathrm{m}}\right)$ of WT DLL4 was $47^{\circ} \mathrm{C}$ and that the $\mathrm{T}_{\mathrm{m}}$ of Delta ${ }^{\mathrm{MAX}}$ was increased by $11^{\circ} \mathrm{C}$ to $58^{\circ} \mathrm{C}$ (Fig. 189 2d). Furthermore, expression yield of the Delta ${ }^{\mathrm{MAX}}$ protein was increased by 2-fold compared to 190 WT DLL4 (Extended Data Fig. 4g). Taken together, our SPR and DSF measurements indicate that 191 Delta $^{\mathrm{MAX}}$ exhibits enhanced affinity, stability and expression while maintaining a broad reactivity 192 profile against human and murine Notch receptors.

194 Delta ${ }^{\text {MAX }}$ activates Notch more potently than WT DLL4. We performed a series of signaling assays to evaluate the Notch activation potency, signaling kinetics, and cross-reactivity of our high-affinity Delta ${ }^{\mathrm{MAX}}$ ligand. We first characterized the signaling potency of Delta ${ }^{\mathrm{MAX}}$ protein using fluorescent (H2B-Citrine) Notch1 reporter cells (Extended Data Fig. 6a, 7a) ${ }^{31}$. As our goal was to develop an accessible tool for modulating Notch activity, we performed this assay by nonspecifically adsorbing DLL4 proteins to 96-well tissue culture plates. This facile method requires minimal manipulation of proteins and is an established method for stimulating Notch activation in vitro. Plates were coated with various concentrations of WT DLL4 or Delta ${ }^{\mathrm{MAX}}$ and Notch1 reporter cells were cultured on the ligand-coated surfaces. After 24 hours, fluorescence was monitored by flow cytometry. Fitting the data revealed that EC50 values for Notch1 activation by WT DLL4 and Delta ${ }^{\mathrm{MAX}}$ were $1.7 \mu \mathrm{M}$ and $6.8 \mathrm{nM}$, respectively, which corresponds to a 250-fold improvement in Notch activation efficiency (Fig. 3a). Moreover, the $\mathrm{E}_{\max }$ induced by Delta ${ }^{\mathrm{MAX}}$ was $20 \%$ greater than that induced by WT DLL4, indicating that Delta ${ }^{\mathrm{MAX}}$ is capable of activating Notch signaling with greater amplitude and potency than WT DLL4.

We next tested whether C-terminal anchoring of DLL4 proteins improves signaling output. This 210 strategy mimics the orientation the proteins adopt on the cell surface and was achieved by 211 biotinylating the ligand C-termini prior to attachment to streptavidin-coated plates. For both WT 212 DLL4 and Delta ${ }^{\mathrm{MAX}}$, oriented coupling induced a higher maximum level of Notch1 activation than 213 non-specific adsorption (Fig. 3b). In this format, Delta ${ }^{\mathrm{MAX}}$ was also more potent than WT DLL4 214 and activated Notch1 with an EC50 of $1.7 \mathrm{nM}$ compared to $16 \mathrm{nM}$ for WT DLL4. We also 
215 performed a time-course experiment to evaluate the signaling kinetics of Delta ${ }^{\mathrm{MAX}}$. We determined 216 that Delta ${ }^{\mathrm{MAX}}$ activates Notch1 more rapidly than WT DLL4, and that this effect is most 217 pronounced in the first 4 to 8 hours following ligand stimulation (Fig. 3c). These findings indicate 218 that the high-affinity Delta ${ }^{\mathrm{MAX}}$ ligand potently activates Notch regardless of coupling strategy, and 219 that oriented coupling maximizes the efficiency of Notch activation through DLL4 ligands.

Delta $^{\mathrm{MAX}}$ strongly activates multiple human Notch receptors. We tested the ability of Delta ${ }^{\mathrm{MAX}}$

222 to activate different human Notch receptor subtypes using an established luciferase reporter assay.

223 For this assay, we used U2OS cells stably expressing chimeric Notch1, Notch2, or Notch3 proteins

224 (Extended Data Fig. 6b) ${ }^{32}$. Notch4 was excluded from our study since it has been suggested that

225 Notch4 inhibits signaling and it is not responsive to ligand-mediated activation in vitro ${ }^{33}$. To

226 compare the signaling of each ligand, we cultured Notch1, Notch2, and Notch3 reporter cells with

227 C-terminally anchored WT DLL4 or high-affinity Delta ${ }^{\text {MAX }}$ (Fig 3d). When we stimulated Notch1

228 cells with WT DLL4, we observed a dose-dependent increase in Notch signaling. The Delta ${ }^{\mathrm{MAX}}$

229 variant more potently activated Notch1 across all concentrations and induced 2-fold higher levels

230 of Notch1 signaling relative to WT DLL4 at the highest concentration tested (50 nM). Delta ${ }^{\text {MAX }}$

231 also activated Notch2 and Notch3 more potently than WT DLL4. For Notch2 and Notch3, relative

232 Delta ${ }^{\mathrm{MAX}}$ signaling was increased by 2.5-fold and 3-fold, respectively. These functional data

233 indicate that enhanced affinity of Delta ${ }^{\mathrm{MAX}}$ leads to increased signaling through Notch1-3, and that

234 Delta $^{\mathrm{MAX}}$ is a viable tool for potently activating multiple human Notch receptors.

236 To determine whether Delta ${ }^{\mathrm{MAX}}$ stimulates increased activation in cells expressing endogenous 237 levels of Notch1, we compared the signaling of Delta ${ }^{\mathrm{MAX}}$ and WT DLL4 in U2OS (osteosarcoma 238 epithelial) cells and MCF-7 (breast cancer). U2OS and MCF-7 cells were each stimulated for 24 239 hours using C-terminally anchored WT DLL4 and Delta ${ }^{\text {MAX }}$ and Notch1 activation was detected 240 by Western blotting for the cleaved Notch1 ICD (Fig. 3e). We found that Delta ${ }^{\text {MAX }}$ activated Notch 241 signaling much more potently than WT DLL4 in both cell lines, indicating that Delta ${ }^{\mathrm{MAX}}$ is a more 242 potent ligand regardless of Notch expression level.

244 Thus far, all our signaling assays were performed using purified recombinant ligands. To evaluate 245 the potency of Delta ${ }^{\mathrm{MAX}}$ when expressed as a full-length transmembrane protein in cells, we 
performed a co-culture Notch signaling assay. We generated HEK293T cell lines stably expressing either full-length WT DLL4 or Delta ${ }^{\mathrm{MAX}}$ (Extended Data Fig. 7b) and then co-cultured the ligandexpressing cells with Notch1, Notch2, or Notch3 reporter cells (Extended Data Fig. 6b) at a 1:1 ratio. In this co-culture format, we determined that the $\mathrm{E}_{\max }$ of Delta ${ }^{\mathrm{MAX}}$ signaling through Notch1 and Notch3 was $\sim 2$-fold higher than WT DLL4. The luciferase signal for Notch2 was low for both ligands, although only Delta ${ }^{\mathrm{MAX}}$ induced a significant level of Notch2 activation compared to unstimulated cells $(P=0.037)$ (Fig. 3f).

254 Comparison of Delta ${ }^{\mathrm{MAX}}$ signaling on plates, beads, and cells. A myriad of ligand-presentation

255 platforms have been developed to activate mechanosensitive Notch receptors, including ligand256 coated plates, ligand-coated beads, antibody-clustered ligands, ligand-infused matrices, and ligand-overexpressing stromal cell lines ${ }^{13,14,18,30,34-36}$. To our knowledge, no single experiment has directly compared the level of Notch signaling induced across different ligand-presentation formats. Here, we compared the signaling of Delta ${ }^{\mathrm{MAX}}$ in five different formats to determine an optimal strategy for maximizing Notch activation. Fluorescent Notch1 reporter cells were cultured with yeast-displayed Delta ${ }^{\mathrm{MAX}}$, $293 \mathrm{~T}$ cells-expressing Delta $^{\mathrm{MAX}}$, Delta ${ }^{\mathrm{MAX}}$-coated microbeads, plates coated adsorbed with Delta ${ }^{\mathrm{MAX}}$ (non-specific coupling), and streptavidin plates coated with 263 C-terminally biotinylated Delta ${ }^{\mathrm{MAX}}$ (oriented coupling) (Fig. 3g, Extended Data Fig. 8). We 264 determined that yeast-displayed Delta ${ }^{\mathrm{MAX}}$ did not induce a significant change in Notch1 activation, which may be due to the inability of cell wall-tethered ligands to undergo endocytosis, or because the low mass of the yeast cells provides insufficient resistance. However, the remaining formats induced 15- to 50-fold increases in Notch1 activation relative to controls. The co-culture method induced the lowest level of Notch1 activation (15-fold), followed by Delta ${ }^{\text {MAX }}$-coated beads (21-

269 fold), non-specific adsorption (23-fold), and oriented coupling (50-fold) (Fig. 3g). Taken together,

270 our data reveal that recombinant protein-based formats (plate- or bead-bound ligands) induced

271 higher levels signaling than cell-based methods. Furthermore, the increased $\mathrm{E}_{\max }$ of C-terminally 272 anchored Delta ${ }^{\mathrm{MAX}}$ suggests that the greater accessibility of the ligand-binding C2-EGF3 domains 273 is optimal for maximal Notch stimulation.

275 Soluble Delta ${ }^{\text {MAX }}$ is a potent pan-Notch inhibitor. In the absence of membrane anchor, DLL4 
277 However, WT DLL4 binds to human Notch receptors with micromolar affinities that fall outside 278 of the expected range for effective pharmacological inhibition. We hypothesized that our high279 affinity DLL4 variants would overcome the affinity-limitations of WT DLL4 to function as potent 280 Notch antagonists. To test this hypothesis, we cultured fluorescent Notch1 reporter cells on C281 terminally anchored WT DLL4-coated plates in the presence of soluble competitors. We added 282 increasing concentrations of soluble WT DLL4, DLL4.v1, DLL4.v2, and Delta ${ }^{\mathrm{MAX}}$ ECDs to the 283 cells and then monitored reporter signal after 24 hours. We determined that, among the four 284 proteins, only soluble Delta ${ }^{\mathrm{MAX}}$ was able to efficiently block plate-bound WT DLL4-induced 285 Notch1 activation and inhibited signaling with an IC50 of $0.6 \mathrm{nM}$. By contrast, administration of 286 soluble WT DLL4 only reduced reporter activity by $25 \%$ at the highest concentration tested (300 $287 \mathrm{nM}$ ). DLL4.v1 and DLL4.v2 were more potent inhibitors than WT DLL4, but less potent than Delta $^{\mathrm{MAX}}$, and neither DLL4.v1 or DLL4.v2 fully blocked Notch1 activation (Fig. 4a).

Most small molecule Notch inhibitors are non-specific and target multifunctional ADAM10/17 or gamma-secretase proteases required for Notch activation. Therefore, we compared the inhibition potency of our Notch-selective Delta ${ }^{\mathrm{MAX}}$ protein to the established gamma-secretase inhibitor DAPT and BB-94, a broad-spectrum metalloprotease inhibitor, BB-94 that inhibits ADAM10 (Fig. 4b). We determined that Delta ${ }^{\mathrm{MAX}}$ inhibited Notch1 activation with an IC50 of $1 \mathrm{nM}$, which was 40-fold more potent than DAPT (IC50 of $40 \mathrm{nM}$ ) and 1000-fold more potent than BB-94 (IC50 of $1000 \mathrm{nM})$. We also compared the inhibition of Delta ${ }^{\mathrm{MAX}}$ and DAPT in a co-culture assay. Both DAPT and Delta ${ }^{\mathrm{MAX}}$ inhibited cellular DLL4 less potently than plate-bound DLL4, and the IC50 of DAPT and Delta ${ }^{\mathrm{MAX}}$ were 100 and $400 \mathrm{nM}$, respectively (Extended Data Fig. 9). Thus, Delta ${ }^{\mathrm{MAX}}$ functions as a potent and selective inhibitor of ligand-mediated Notch signaling, and the potency of inhibition depends on the format in which the wild-type ligand is presented.

We also tested the ability of Delta ${ }^{\mathrm{MAX}}$ to inhibit signaling by ligands other than DLL4. First, we generated stable HEK293T cell lines expressing DLL1, DLL4, Jag1, and Jag2 and confirmed

304 ligand-expression levels by antibody staining (Extended Data Fig. 10). We then co-cultured DLL1, 305 DLL4, Jag1 or Jag2 cells with Notch3 luciferase reporter cells that were pre-mixed with a constant $306(3 \mu \mathrm{M})$ concentration of soluble WT DLL4 or Delta ${ }^{\mathrm{MAX}}$ (Fig. 4c). We determined that Delta ${ }^{\mathrm{MAX}}$ 307 effectively inhibited productive signaling by DLL1, DLL4, Jag1, and Jag2, with a reduction in 
reporter signal ranging from $70-85 \%$. On the other hand, WT DLL4 only weakly inhibited Notch activation through the four ligands, reducing reporter signal by $10-40 \%$.

Lastly, we assessed whether Delta ${ }^{\mathrm{MAX}}$ could inhibit signaling through different human Notch

312 paralogs. We incubated Notch1, Notch2 and Notch3 luciferase reporter cell lines with various

313 concentrations of soluble WT DLL4 or Delta ${ }^{\mathrm{MAX}}$, and then cultured the cells on plates coated with

314 DLL4-coated plates to monitor Notch activation (Fig. 4d). We determined that addition of the

315 Delta $^{\mathrm{MAX}}$ competitor led to dose-dependent decreases in signaling for Notch1, Notch2, and

316 Notch3, and that WT DLL4 did not significantly inhibit Notch activation at all concentrations

317 tested.

Delta $^{\mathrm{MAX}}$ increased human $\mathrm{CD8}^{+} \mathbf{T}$ cell proliferation and effector markers. Notch signaling contributes to several aspects of immunobiology and influences the proliferation, differentiation, and antitumor function of $\mathrm{CD}^{+} \mathrm{T}$ cells ${ }^{37-41}$. Therefore, we developed an assay to test the effect of Delta $^{\mathrm{MAX}}$ on activated T cells (Fig. 5a). As stromal cells are an established platform for Notch stimulation in T cells, we generated artificial antigen presenting K562 cells (aAPCs) expressing the Fc receptor CD32 (renamed K32 cells), and similar surface levels of WT DLL4 or Delta ${ }^{\mathrm{MAX}}$ (Fig. 5b $)^{42}$. We then co-cultured these ligand-expressing cells with primary $\mathrm{T}$ cells in the presence of increasing concentrations of the T cell-activating antibody OKT3. CD32 expression enabled OKT3 Fc binding to K32 cells and subsequent CD3-TCR activation on T cells. Human CD8 ${ }^{+} \mathrm{T}$ cells were negatively isolated from peripheral blood and co-cultured with K32 cells pre-loaded with increasing concentrations of OKT3 to analyze T cell proliferation and IFN $\gamma$ secretion (Fig. $5 \mathrm{c}, 5 \mathrm{~d})$. Delta ${ }^{\mathrm{MAX}}$ treatment enhanced the percentage of proliferating $\mathrm{CD}^{+} \mathrm{T}$ cells between $27 \%$ and $15 \%$ along the OKT3 concentrations assayed $(P=0.032)$, while WT DLL4 did not show a

332 significant improvement over the stimulation with K32 cells lacking DLL4 ligands (mock) (Fig.

333 5c). Additionally, Delta ${ }^{\mathrm{MAX}}$ increased IFN $\gamma$ secretion from $\mathrm{CD}^{+} \mathrm{T}$ cells $10-15 \%$ compared with

334 WT DLL4 treatment $(P=0.047)$ (Fig. 5d).

336 Next, we used RT-PCR to quantify the mRNA levels of Notch and T cell effector related markers over four days of $\mathrm{CD}^{+} \mathrm{T}$ cell stimulation with $\mathrm{K} 32$ loaded with $0.0125 \mu \mathrm{g} / \mathrm{mL}$ OKT3. We 
Granzyme B, as well as elevated expression of Notch target Hes-4 compared to counterparts conditioned with WT DLL4 or mock (Fig. 5e). Lastly, we performed a western blot analysis to compare Notch 1 and Notch2 activation in T cells that were stimulated with WT DLL4 or Delta ${ }^{\mathrm{MAX}}$.

342 Using an antibody to the cleaved Notch1 or Notch2 ICD, we determined that activation of both

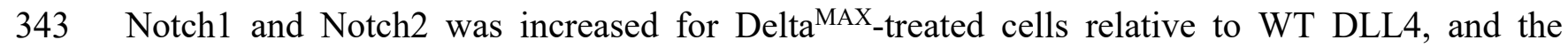
344 difference in Notch2 activation was more prominent than that of Notch1 (Fig. 5f). Thus, results 345 show the promotion of $\mathrm{CD}^{+} \mathrm{T}$ cell function induced by aAPCs expressing Delta ${ }^{\mathrm{MAX}}$.

\section{DISCUSSION}

348 Recombinant DLL and Jag ligand ECDs bind weakly to Notch receptors and are inefficient modulators of Notch signaling. We engineered the synthetic Delta ${ }^{\mathrm{MAX}}$ ligand to be a versatile tool for activating or inhibiting mammalian Notch receptors, depending on the manner in which it is administered. Compared to WT DLL4, immobilized or cellular Delta ${ }^{\mathrm{MAX}}$ exhibits varying degrees of increased signaling potency, and soluble Delta ${ }^{\mathrm{MAX}}$ functioned as a Notch-specific inhibitor when administered as a soluble decoy. Furthermore, the increased thermostability $\left(\Delta \mathrm{T}_{\mathrm{m}} 11^{\circ} \mathrm{C}\right)$ and expression (2-fold higher yield) of Delta ${ }^{\mathrm{MAX}}$ are both favorable properties for protein biologics.

356 Our functional studies revealed that Delta ${ }^{\mathrm{MAX}}$ activates Notch more potently than WT DLL4 in 357 plate-bound, bead-bound, and cellular formats. Among the methods tested, we determined that Cterminally anchored Delta ${ }^{\mathrm{MAX}}$ induced the highest levels of Notch signaling, followed by nonspecifically adsorbed ligands, ligand-coated beads, and cellular ligands. The largest difference in signaling potency between WT DLL4 and Delta ${ }^{\mathrm{MAX}}$ was observed when ligands were nonspecifically adsorbed to plastic. The WT DLL4 protein was a poor Notch activator in this format (EC50 $1.7 \mu \mathrm{M}$ ), while Delta ${ }^{\mathrm{MAX}}$ was remarkably potent (EC50 $6.8 \mathrm{nM}$ ). The strong signaling activity can enable entire flasks or tissue culture plates to be pre-conditioned with Delta ${ }^{\mathrm{MAX}}$ as a high-throughput means of activating Notch in cultured cells. Furthermore, non-specific adsorption requires low quantities of protein and can be performed with unmodified ligand ECDs, making this method highly accessible for a wide range of biotechnological applications.

368 Soluble DLL4 ECDs do not activate Notch because they are unable to exert the requisite pulling 
site on the Notch receptor. We demonstrated that, even at high-concentrations, the micromolar binding affinity of WT DLL4 prevents it from functioning as an effective Notch antagonist. On the other hand, Delta ${ }^{\mathrm{MAX}}$ potently inhibited Notch activation by all four activating ligands (DLL1, DLL4, Jag1, and Jag2). The broad-reactivity profile of Delta ${ }^{\mathrm{MAX}}$ also enabled it to inhibit ligandmediated activation of Notch1, Notch2, and Notch3. Current strategies for pan-Notch inhibition require the use of gamma-secretase or ADAM10/17 inhibitors, which block the cleavage of numerous cellular substrates in addition to Notch. Alternatively, antibodies targeting the NRR domain have been used to block signaling by individual Notch receptor subtypes. We found that Delta $^{\mathrm{MAX}}$ inhibited Notch signaling with similar potency to the established gamma-secretase inhibitor DAPT. Therefore, this ability of Delta ${ }^{\mathrm{MAX}}$ to block signaling by all Notch receptors makes it a unique pharmacological tool as a highly selective, pan-Notch inhibitor.

Notch signaling is central regulator of $\mathrm{T}$ cell biology and influences $\mathrm{T}$ cell activation, proliferation, and cytolytic effector functions ${ }^{37-40,43,44}$. We previously demonstrated that constitutive Notch activation by overexpression of NICD enhances the antitumor activity of adoptively transferred murine $\mathrm{CD}^{+} \mathrm{T}$ cells ${ }^{45}$. As this method required genetic modification of the $\mathrm{T}$ cells, we aimed to develop an ex vivo platform for transient Notch activation using Delta ${ }^{\mathrm{MAX}}$. In the present study, we determined that co-culturing activated T cells with K32 cells expressing Delta ${ }^{\mathrm{MAX}}$ induces increased $\mathrm{T}$ cell proliferation and expression of effector mediators compared to co-culture with WT DLL4. This approach could be potentially beneficial for strategies of expansion of anti-tumor CAR-T or TILS for further adoptive transfer by triggering Notch-related metabolic and anti-tumor signals.

393 Various ligand presentation strategies have been developed for activating Notch in T cells, and in 394 these systems, the dosage of Notch signaling has proven to be important for achieving optimal 395 outcomes. For example, low doses of DLL1 stimulate the differentiation of hematopoietic stem 396 and progenitor cells (HSPCs) into mixed populations of lymphoid and myeloid precursors, 397 whereas high-doses promote the differentiation of only lymphoid precursors ${ }^{46}$. Additionally, 398 stromal cells that express DLL4, which binds to Notch1 with higher affinity than DLL1 ${ }^{13}$, more 399 efficiently drives $\mathrm{T}$ cell lymphopoiesis ${ }^{35}$. Given the tunable outcomes of $\mathrm{T}$ cell behavior, we 
anticipate that the ultra-potent Delta ${ }^{\mathrm{MAX}}$ ligand will be a powerful tool for achieving ideal levels of Notch activation for T cell-related applications.

\section{ACKNOWLEDGMENTS}

404 We would like to thank Dr. Eric Lau, Dr. Gregory Watson and Daniel Lester for their helpful assistance in generating DLL4 stable cell lines. We also thank Dr. Timothy Tran from Moffitt Chemical Biology Core for helping with DSF studies, and Dr. Qianqian Ming for helpful suggestions. D.G. and V.L. were supported by R35GM133482 and an award from the Rita Allen Foundation. S.C.B. is supported by R35CA220340. P.C.R. and S.D. were supported by R01CA184185, R01CA233512; R01CA262121; P01CA250984 Project \#4; and P30CA076292; and Florida Department of Health grant \#20B04. Support for shared resources was provided by the Moffitt Cancer Center Support Grant NIH P30CA076292.

\section{AUTHOR CONTRIBUTION}

414 V.C.L. and D.G.P. wrote the manuscript. V.C.L., D.G.P, D.A., and P.C.R. designed the 415 experiments. D.G.P. performed the protein purifications, yeast display selections, binding studies,

416 and signaling assays. S.D. performed and designed the $T$ cell experiments with assistance from

417 P.C.R. E.M. contributed to library design and cloning. D.A. performed the detection of Notch 418 activation assays on U2OS and MCF-7 cells by Western blot. E.D.E. and S.C.B. provided U2OS 419 reporter cells and assisted with luciferase experimental procedures. S.C.B., P.C.R., and V.C.L. 420 edited and reviewed the manuscript. V.C.L. supervised and conceived the project.

\section{CONFLICT OF INTEREST}

423 V.C.L., P.C.R., and D.G.P., have filed provisional patents describing the engineering and applications of Delta $^{\text {MAX }}$ (application numbers PCT/US2020/041765 and PCT/US2020/030977). V.C.L. is a consultant on an unrelated project for Cellestia Biotech. S.C.B. is on the SAB and receives funding from ERASCA, Inc. for an unrelated project, and is a consultant on unrelated projects for Scorpion Therapeutics, Odyssey Therapeutics, Ayala Pharmaceuticals, MPM Capital, and Droia Ventures. 


\section{REFERENCES}

1. Sprinzak, D. \& Blacklow, S. C. Biophysics of Notch Signaling. Annu. Rev. Biophys. 50, 157-189 (2021).

2. Kopan, R. \& Ilagan, Ma. X. G. The Canonical Notch Signaling Pathway: Unfolding the Activation Mechanism. Cell 137, 216-233 (2009).

3. Lovendahl, K. N., Blacklow, S. C. \& Gordon, W. R. The Molecular Mechanism of Notch Activation. in Molecular Mechanisms of Notch Signaling (eds. Borggrefe, T. \& Giaimo, B. D.) vol. 1066 47-58 (Springer International Publishing, 2018).

4. Cordle, J. et al. A conserved face of the Jagged/Serrate DSL domain is involved in Notch trans-activation and cis-inhibition. Nature Structural \& Molecular Biology 15, 849-857 (2008).

5. Gordon, W. R. et al. Mechanical Allostery: Evidence for a Force Requirement in the Proteolytic Activation of Notch. Developmental Cell 33, 729-736 (2015).

6. Gordon, W. R. et al. Structural basis for autoinhibition of Notch. Nat Struct Mol Biol 14, 295-300 (2007).

7. Parks, A. L., Klueg, K. M., Stout, J. R. \& Muskavitch, M. A. Ligand endocytosis drives receptor dissociation and activation in the Notch pathway. Development 127, 1373-1385 (2000).

8. Seib, E. \& Klein, T. The role of ligand endocytosis in notch signalling. Biol. Cell 113, 401418 (2021).

9. Mumm, J. S. et al. A Ligand-Induced Extracellular Cleavage Regulates $\gamma$-Secretase-like Proteolytic Activation of Notch1. Molecular Cell 5, 197-206 (2000).

10. De Strooper, B. et al. A presenilin-1-dependent $\gamma$-secretase-like protease mediates release of Notch intracellular domain. Nature 398, 518-522 (1999).

11. Wu, L. et al. MAML1, a human homologue of Drosophila Mastermind, is a transcriptional co-activator for NOTCH receptors. Nat Genet 26, 484-489 (2000).

12. Yamamoto, S. et al. A Mutation in EGF Repeat-8 of Notch Discriminates Between Serrate/Jagged and Delta Family Ligands. Science 338, 1229-1232 (2012).

13. Andrawes, M. B. et al. Intrinsic selectivity of Notch 1 for Delta-like 4 over Delta-like 1. J. Biol. Chem. 288, 25477-25489 (2013). 
14. Luca, V. C. et al. Structural basis for Notch1 engagement of Delta-like 4. Science 347, 847$853(2015)$.

15. Luca, V. C. et al. Notch-Jagged complex structure implicates a catch bond in tuning ligand sensitivity. Science eaaf9739 (2017) doi:10.1126/science.aaf9739.

16. Tveriakhina, L. et al. The ectodomains determine ligand function in vivo and selectivity of DLL1 and DLL4 toward NOTCH1 and NOTCH2 in vitro. Elife 7, e40045 (2018).

17. Hicks, C. et al. A secreted Delta1-Fc fusion protein functions both as an activator and inhibitor of Notch1 signaling. J. Neurosci. Res. 68, 655-667 (2002).

18. Biktasova, A. K. et al. Multivalent Forms of the Notch Ligand DLL-1 Enhance Antitumor Tcell Immunity in Lung Cancer and Improve Efficacy of EGFR-Targeted Therapy. Cancer Res 75, 4728-4741 (2015).

19. Nandagopal, N. et al. Dynamic Ligand Discrimination in the Notch Signaling Pathway. Cell 172, 869-880.e19 (2018).

20. Benedito, R. et al. The notch ligands Dll4 and Jagged1 have opposing effects on angiogenesis. Cell 137, 1124-1135 (2009).

21. Van de Walle, I. et al. Specific Notch receptor-ligand interactions control human TCR- $\alpha \beta / \gamma \delta$ development by inducing differential Notch signal strength. Journal of Experimental Medicine 210, 683-697 (2013).

22. Nowell, C. S. \& Radtke, F. Notch as a tumour suppressor. Nature Reviews Cancer 17, 145159 (2017).

23. Ran, Y. et al. $\gamma$-Secretase inhibitors in cancer clinical trials are pharmacologically and functionally distinct. EMBO Mol Med 9, 950-966 (2017).

24. Goruganthu, M. U. L., Shanker, A., Dikov, M. M. \& Carbone, D. P. Specific Targeting of Notch Ligand-Receptor Interactions to Modulate Immune Responses: A Review of Clinical and Preclinical Findings. Front. Immunol. 11, 1958 (2020).

25. Espinoza, I. \& Miele, L. Notch inhibitors for cancer treatment. Pharmacology \& Therapeutics 139, 95-110 (2013).

26. Tiyanont, K., Wales, T. E., Siebel, C. W., Engen, J. R. \& Blacklow, S. C. Insights into Notch3 Activation and Inhibition Mediated by Antibodies Directed against Its Negative Regulatory Region. Journal of Molecular Biology 425, 3192-3204 (2013). 
27. Xu, X. et al. Insights into Autoregulation of Notch3 from Structural and Functional Studies of Its Negative Regulatory Region. Structure 23, 1227-1235 (2015).

28. Güner, G. \& Lichtenthaler, S. F. The substrate repertoire of $\gamma$-secretase/presenilin. Seminars in Cell \& Developmental Biology 105, 27-42 (2020).

29. Komatsu, H. et al. OSM-11 Facilitates LIN-12 Notch Signaling during Caenorhabditis elegans Vulval Development. PLoS Biol 6, e196 (2008).

30. Liu, L., Wada, H., Matsubara, N., Hozumi, K. \& Itoh, M. Identification of Domains for Efficient Notch Signaling Activity in Immobilized Notch Ligand Proteins: CRITICAL DOMAINS OF IMMOBILIZED LIGANDS FOR NOTCH S IGNALING. J. Cell. Biochem. 118, 785-796 (2017).

31. Sprinzak, D. et al. Cis-interactions between Notch and Delta generate mutually exclusive signalling states. Nature 465, 86-90 (2010).

32. Malecki, M. J. et al. Leukemia-Associated Mutations within the NOTCH1 Heterodimerization Domain Fall into at Least Two Distinct Mechanistic Classes. Mol Cell Biol 26, 4642-4651 (2006).

33. James, A. C. et al. Notch4 reveals a novel mechanism regulating Notch signal transduction. Biochimica et Biophysica Acta (BBA) - Molecular Cell Research 1843, 1272-1284 (2014).

34. Trotman-Grant, A. C. et al. DL4- $\mu$ beads induce T cell lineage differentiation from stem cells in a stromal cell-free system. Nat Commun 12, 5023 (2021).

35. Mohtashami, M. et al. Direct Comparison of D111- and D114-Mediated Notch Activation Levels Shows Differential Lymphomyeloid Lineage Commitment Outcomes. The Journal of Immunology 185, 867-876 (2010).

36. Safaee, H. et al. Tethered Jagged-1 Synergizes with Culture Substrate Stiffness to Modulate

37. Bheeshmachar, G. et al. Evidence for a Role for Notch Signaling in the Cytokine-Dependent Survival of Activated T Cells. J Immunol 177, 5041-5050 (2006).

38. Cho, O. H. et al. Notch regulates cytolytic effector function in CD8+ T cells. J Immunol 182, 3380-3389 (2009).

39. Palaga, T., Miele, L., Golde, T. E. \& Osborne, B. A. TCR-Mediated Notch Signaling 3024 (2003). 
40. Adler, S. H. et al. Notch Signaling Augments T Cell Responsiveness by Enhancing CD25 Expression. J Immunol 171, 2896-2903 (2003).

41. Joshi, I. et al. Notch signaling mediates G1/S cell-cycle progression in T cells via cyclin D3 and its dependent kinases. Blood 113, 1689-1698 (2009).

42. Thomas, A. K., Maus, M. V., Shalaby, W. S., June, C. H. \& Riley, J. L. A Cell-Based Artificial Antigen-Presenting Cell Coated with Anti-CD3 and CD28 Antibodies Enables Rapid Expansion and Long-Term Growth of CD4 T Lymphocytes. Clinical Immunology 105, 259-272 (2002).

43. Eagar, T. N. et al. Notch 1 Signaling Regulates Peripheral T Cell Activation. Immunity 20, 407-415 (2004).

44. Backer, R. A. et al. A central role for Notch in effector CD8(+) T cell differentiation. Nat Immunol 15, 1143-1151 (2014).

45. Sierra, R. A. et al. Rescue of Notch-1 Signaling in Antigen-Specific CD8 ${ }^{+}$T Cells Overcomes Tumor-Induced T-cell Suppression and Enhances Immunotherapy in Cancer. Cancer Immunol Res 2, 800-811 (2014).

46. Delaney, C., Varnum-Finney, B., Aoyama, K., Brashem-Stein, C. \& Bernstein, I. D. Dosedependent effects of the Notch ligand Deltal on ex vivo differentiation and in vivo marrow repopulating ability of cord blood cells. Blood 106, 2693-2699 (2005). 

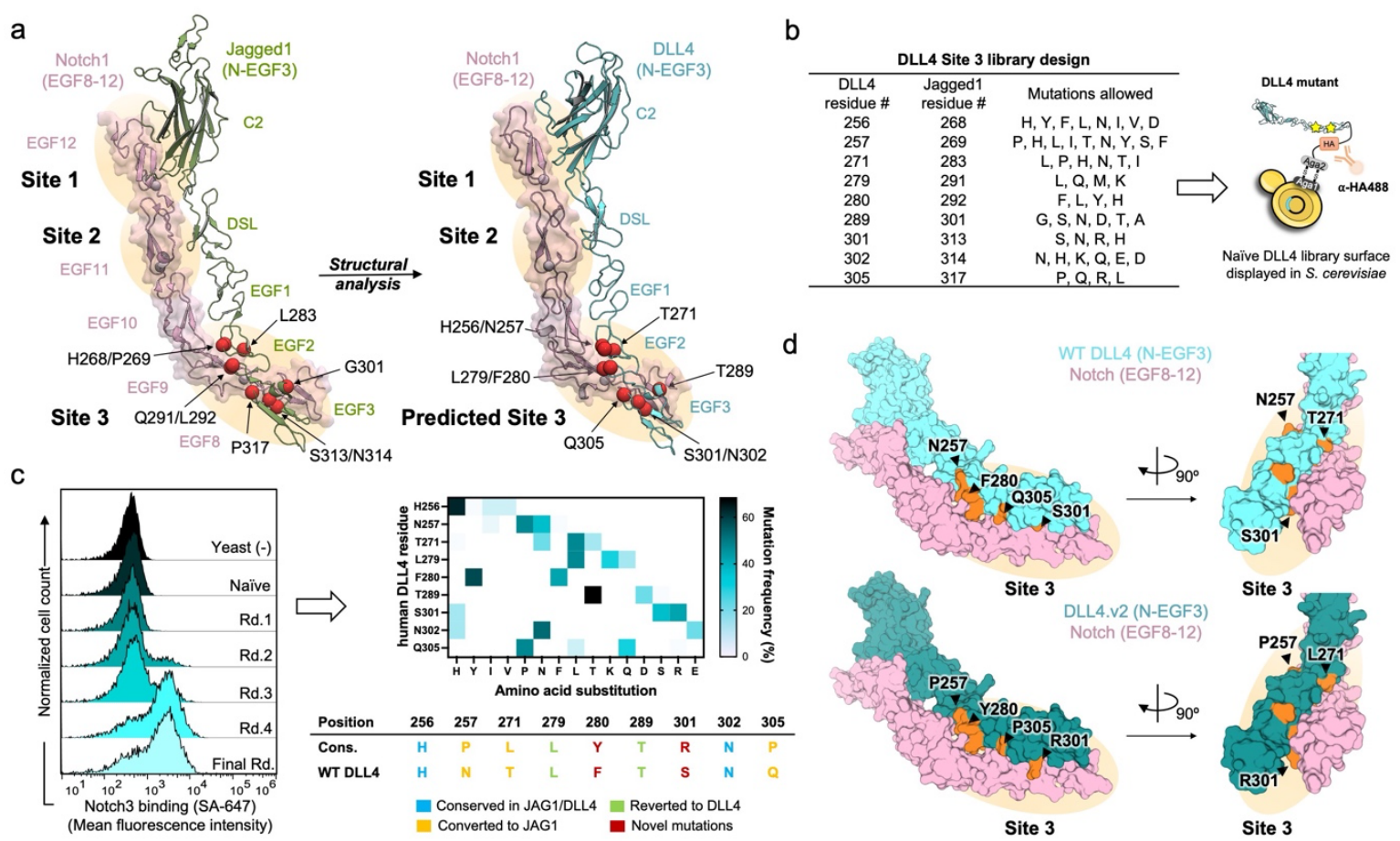

Fig 1. Yeast display selection of affinity-enhancing mutations in DLL4. (A) Cartoon schematic of the design strategy for the DLL4 Site 3 mutant library. Red spheres depict mutated interface residues. (B) Table depicting DLL4 interface residue positions and the mutations allowed at each position and schematic of DLL4 yeast display construct. Yellow stars indicate mutations. (C) Flow cytometry histogram plots of yeast stained with fluorescently-labeled Notch3 protein following each round of selection (left), and a table indicating the frequency of mutations and consensus DLL4 mutant sequence (right). (D) Structural models highlighting the mutated residues in DLL4.v2. WT residues in DLL4 and mutated residues in DLL4.v2 are colored orange and shown in surface representation. DLL4 models were based on DLL4:Notch1 (PDB ID: 4XL1, for C2DSL-EGF1 domains) and Jagged1:Notch1 (PDB ID:5UK5, for EGF1-EGF3) structures. 
a

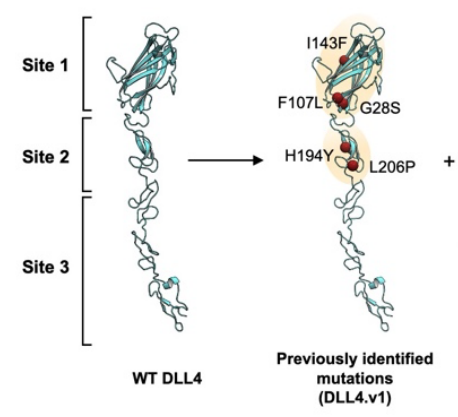

C

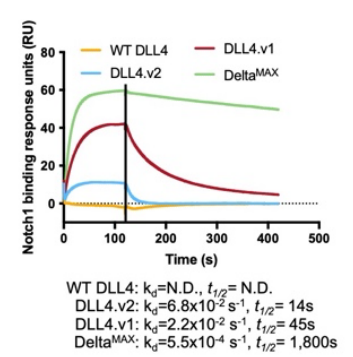

d

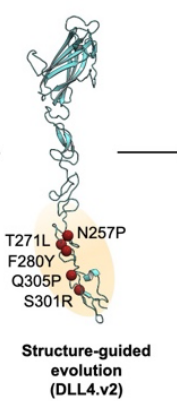

d

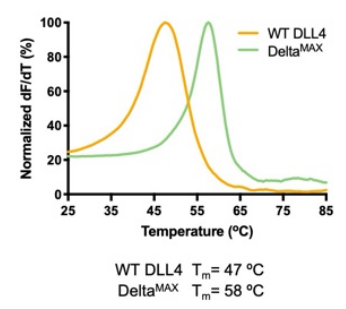

b
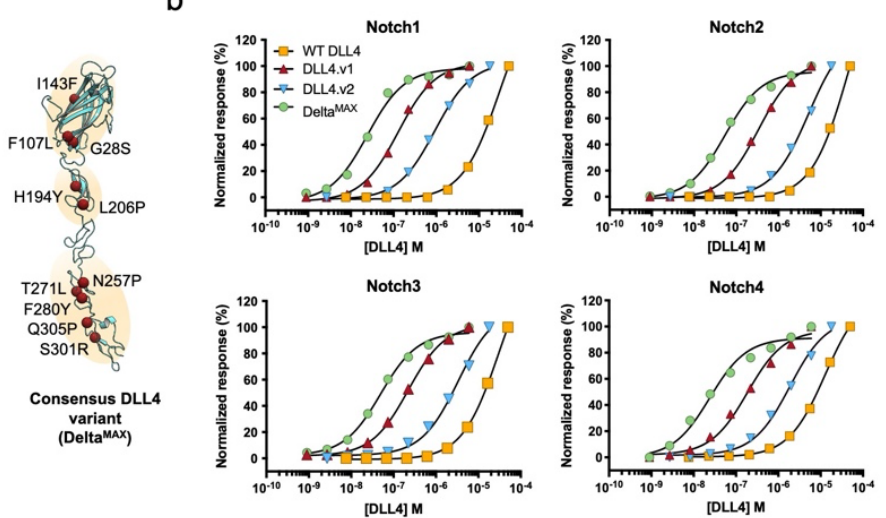

\begin{tabular}{cccccc}
\multicolumn{6}{c}{ Equilibrium dissociation constants (nM) } \\
\hline $\begin{array}{c}\text { Ligand } \\
\text { (N-EGF5) }\end{array}$ & $\begin{array}{c}\text { Notch1 } \\
\text { (EGF6-13) }\end{array}$ & $\begin{array}{c}\text { Notch2 } \\
\text { (EGF6-13) }\end{array}$ & $\begin{array}{c}\text { Notch3 } \\
\text { (EGF5-12) }\end{array}$ & $\begin{array}{c}\text { Notch4 } \\
\text { (EGF7-12) }\end{array}$ & $\begin{array}{c}\text { mNotch1 } \\
\text { (EGF6-13) }\end{array}$ \\
WT DLL4 & 24,700 & 36,200 & 29,000 & 12,770 & 20,600 \\
DLL4.v2 & 870 & 4,830 & 3,330 & 1,877 & 728 \\
DLL4.v1 & 127 & 330 & 221 & 186 & 150 \\
DeltaMAX & 24 & 54 & 49 & 24 & 25 \\
\hline \multicolumn{7}{c}{ Improvement (fold-change) } \\
\hline DeltaMAX & 1,029 & 670 & 591 & 532 & 824 \\
\hline
\end{tabular}

Fig 2. Biophysical characterization of Delta ${ }^{\mathrm{MAX}}$ variant. (A) Schematic depicting the generation of DLL4 ${ }^{\mathrm{MAX}}$ through engraftment of DLL4.v1 and DLL4.v2 mutations. Red spheres indicate affinity-enhancing mutations. (B) SPR binding isotherms measuring DLL4 variant interactions with the ligand binding domains of Notch1-4. The table shows the $K_{D}$ determined for each interaction and the fold-enhancement of DLL4 ${ }^{\mathrm{MAX}}$ affinity relative to WT DLL4. (C) Representative SPR sensograms depicting the binding of $200 \mathrm{nM}$ concentrations of WT DLL4, DLL4.v1, DLL4.v2, and DLL4 ${ }^{\mathrm{MAX}}$ to Notch1 EGF6-13. (D) DSF was used to determine the $\mathrm{T}_{\mathrm{m}}$ of WT DLL4 and Delta ${ }^{\text {MAX }}$. 
a

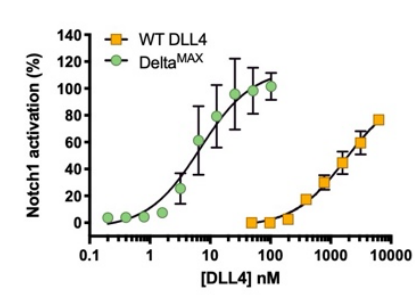

d

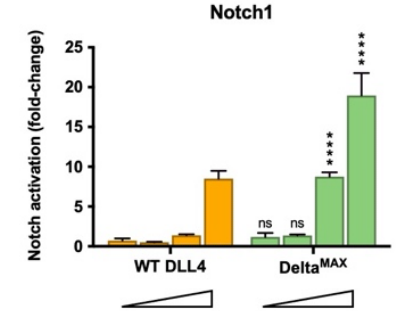

e

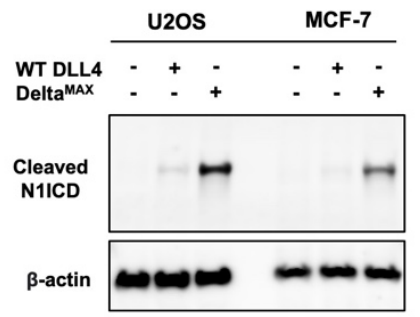

b
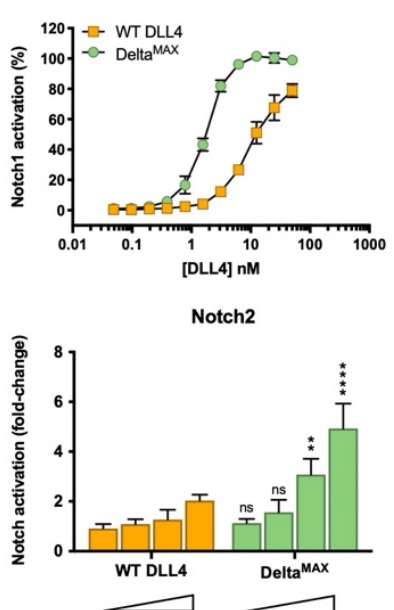

f

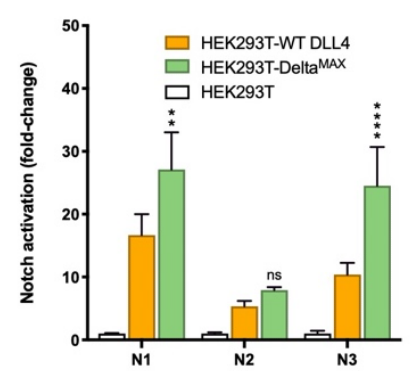

C
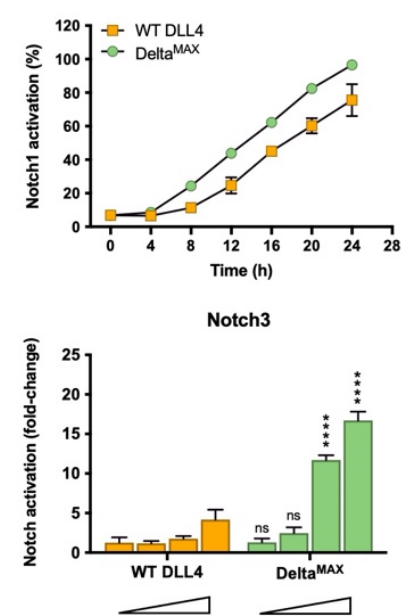

g

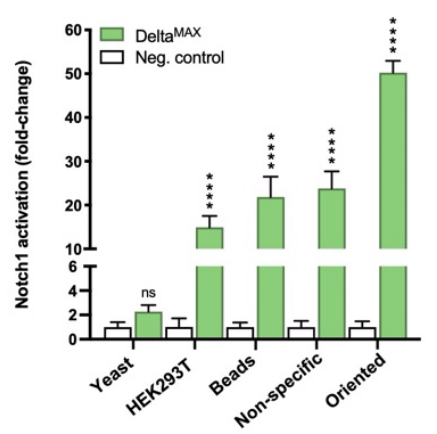

Fig 3. Delta ${ }^{M A X}$ is more potent Notch agonist than WT DLL4. (A) Dose-titration assay comparing the Notch1 reporter activity stimulated by WT DLL4 and Delta ${ }^{\mathrm{MAX}}$ proteins that were non-specifically adsorbed to tissue culture plates. (B) Dose-titration assay comparing the Notch1 reporter activity stimulated by WT DLL4 and Delta ${ }^{\mathrm{MAX}}$ proteins. DLL4 ligands were C-termini biotinylated and coupled to streptavidin-coated plates. (C) Time-course experiment comparing Notch1 reporter activity stimulated by WT DLL4 or Delta ${ }^{\mathrm{MAX}}$ immobilized at $50 \mathrm{nM}$. (D) A luciferase reporter assay was used to measure Notch1, Notch2, or Notch3 activation by WT DLL4 and DLL4 ${ }^{\mathrm{MAX}}$. Protein concentrations were a 10 -fold serial dilution from $50 \mathrm{nM}$ to $0.05 \mathrm{nM}$. (E)

601 Detection of endogenous cleaved Notch intracellular domain (N1ICD) in U2OS and MCF-7 cell 602 lines by Western blot. Cells were Notch activated with $50 \mathrm{nM}$ of WT DLL4 and Delta ${ }^{\mathrm{MAX}}$ 603 immobilized in streptavidin plates. (F) Co-culture assay comparing Notch1 activation by Delta ${ }^{\mathrm{MAX}}$ 604 and WT DLL4. Notch1 reporter cells and ligand-expressing cells were cultured at a 1:1 ratio. (G) 605 Notch1 luciferase reporter cells were stimulated with Delta ${ }^{\mathrm{MAX}}$ protein in different formats. Yeast expressing Delta $^{\mathrm{MAX}}$ were co-cultured in 10:1 ratio with reporter cells; HEK293T cells stably

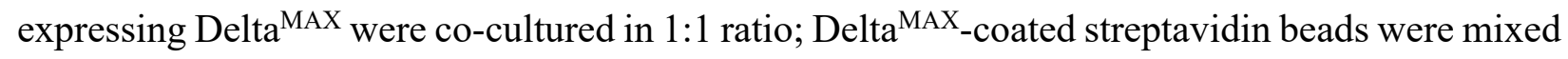
in 20:1 ratio; $100 \mathrm{nM}$ Delta ${ }^{\mathrm{MAX}}$ was non-specifically adsorbed to surfaces prior to addition of 
609 reporter cells; $10 \mathrm{nM}$ C-terminally biotinylated Delta ${ }^{\mathrm{MAX}}$ was immobilized on streptavidin-coated 610 plates. Notch activation was normalized to the corresponding controls. Delta ${ }^{\mathrm{MAX}}$ statistics are 611 referred to WT DLL4. $* \mathrm{P}<0.05, * * \mathrm{P}<0.01$, $* * * * \mathrm{P}<0.0001$, ns: not significant (Two-way 612 ANOVA).

613

614

615

616

617

618

619

620

621

622

623

624

625

626

627

628

629

630

631

632

633

634

635 
a

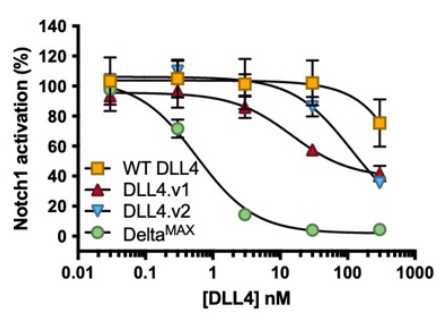

d

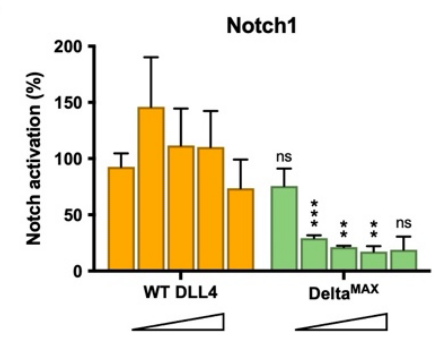

b
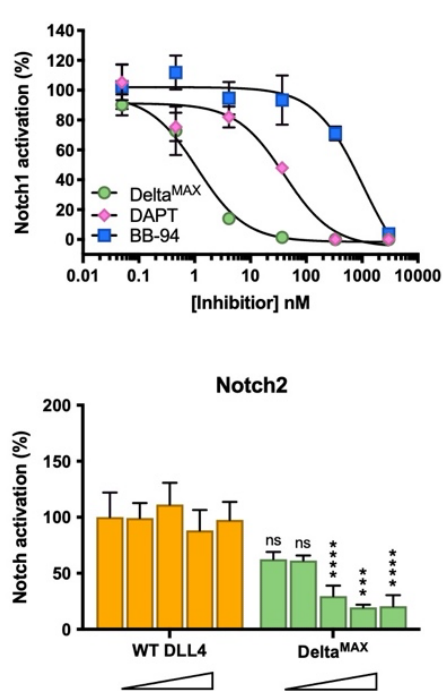

C
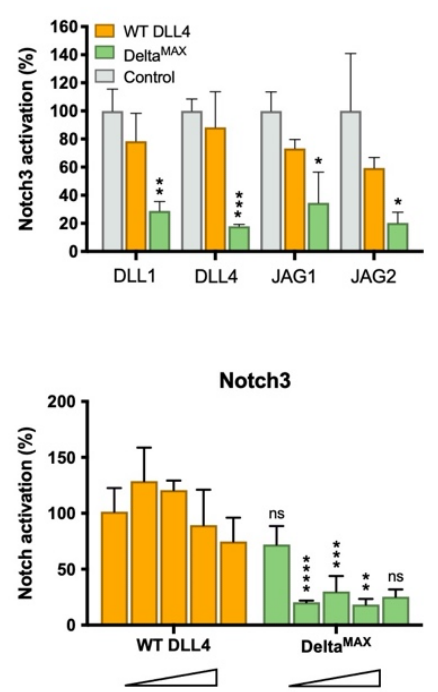

4. Delta ${ }^{\text {MAX }}$ is a pan-Notch inhibitor. (A) Dose-titration assay measuring the inhibition of Notch1 reporter activity by soluble DLL4 variants. Notch1 signaling was activated by culturing cells on streptavidin plates coated with $50 \mathrm{nM}$ WT DLL4. (B) Dose-titration assay comparing the inhibition potency of DAPT, BB-94, and soluble Delta ${ }^{\mathrm{MAX}}$. Notch1 signaling was activated by culturing cells on streptavidin plates coated with 50 nM WT DLL4. (C) Soluble WT DLL4 and Delta ${ }^{\text {MAX }}$ at 3 $\mu \mathrm{M}$ were tested for their ability to inhibit Notch1 activation by $293 \mathrm{~T}$ cells overexpressing DLL4, DLL1, JAG1, or JAG2. (D) Dose-titration assay comparing the ability of WT DLL4 and Delta ${ }^{\mathrm{MAX}}$ to inhibit activation of Notch1-3 by WT DLL4. 10-fold serial dilutions starting at 3,000 nM of soluble DLL4 or Delta ${ }^{\mathrm{MAX}}$ were added to Notch1, Notch2, and Notch3 luciferase reporter cells. Notch signaling was activated by culturing cells on streptavidin plates coated with $50 \mathrm{nM}$ WT DLL4. $* \mathrm{P}<0.05, * * \mathrm{P}<0.01, * * * \mathrm{P}<0.001, * * * * \mathrm{P}<0.0001$, ns: not significant (Two-way ANOVA). 
a

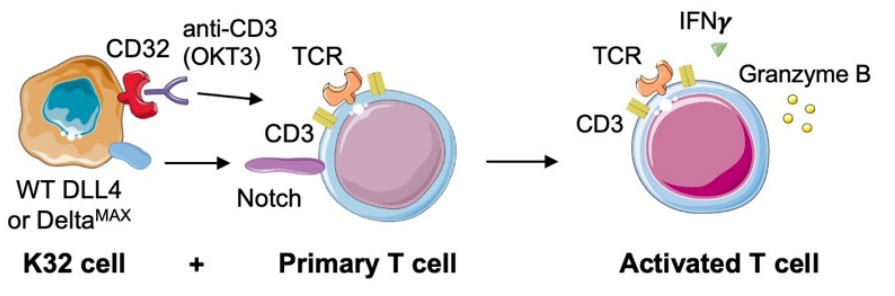

c

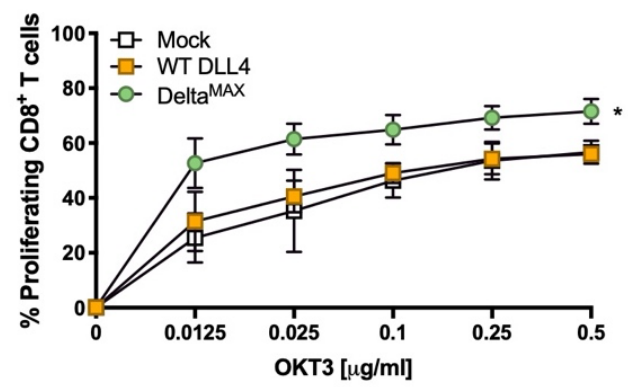

e
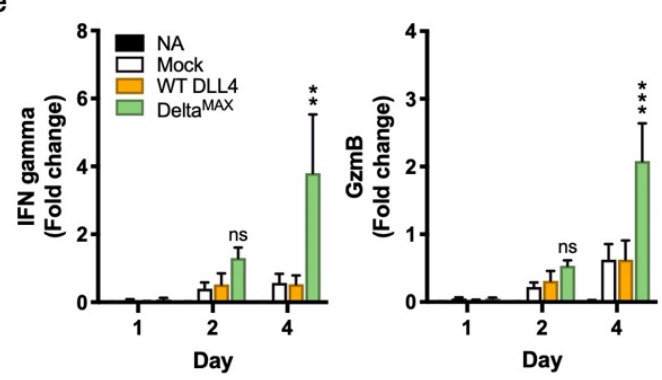

d

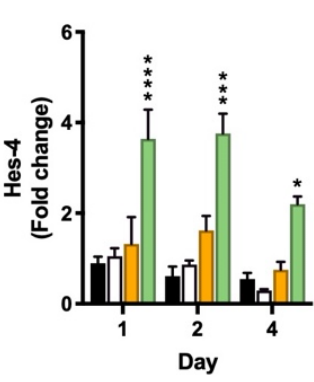

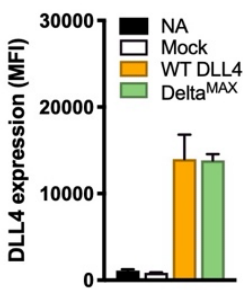

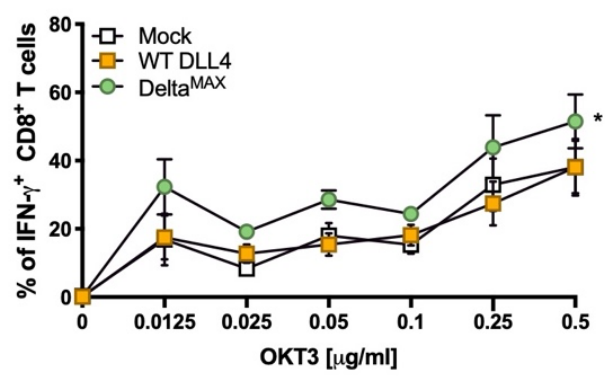

f Co-culture T cells (96h)

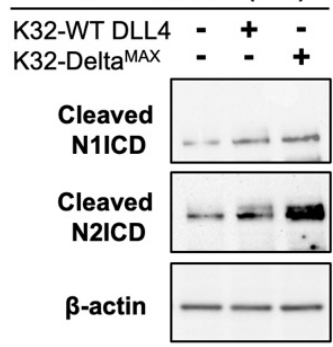

Fig 5. Translational strategy to transiently boost Notch signaling in human $\mathrm{CD8}^{+} \mathrm{T}$ cells. (A) Human PBMCs $\mathrm{CD}^{+} \mathrm{T}$ cells were co-cultured with $\mathrm{K} 32$ artificial antigen-presenting cells (aAPCs) engineered to express WT DLL4 or Delta ${ }^{\mathrm{MAX}}$ for simultaneous Notch and TCR stimulation. (B) aAPCs were assayed for expression of WT DLL4 or Delta ${ }^{\mathrm{MAX}}$ using flow cytometry. K32 cells were loaded with OKT3 antibody using a gradient of concentrations starting at $0.0125 \mu \mathrm{g} / \mathrm{ml}$, and then co-cultured with $\mathrm{CD}^{+} \mathrm{T}$ cells in ratio 10:1. After $96 \mathrm{~h}, \mathrm{CD}^{+} \mathrm{T}$ cells were analyzed for proliferation (C) and IFN $\gamma$ secretion (D). (E) T cells were stimulated for 24, 48, and $96 \mathrm{~h}$ to quantify mRNA levels of intracellular activation markers by real-time PCR, including 666 IFN $\gamma$, Granzyme B and Hes-4. (F) K32 cells expressing WT DLL4 or Delta ${ }^{\mathrm{MAX}}$ were co-culture 667 with human $\mathrm{CD}^{+} \mathrm{T}$ cells (ratio 1:10) for detection of Notch activation by Western blot. Co668 cultured $\mathrm{CD}^{+} \mathrm{T}$ cells were positively sorted by MACS column after cultivation with K32 cells, 669 and nuclear extracts were harvested after 96 hours. Cleavage of Notch1 and Notch2 were examined 670 by Western blot with specific antibodies. $\beta$-actin was used for loading control. Western blot images 671 are representative results of 3 biological repeats. NA: non-activated T cells. Statistics for Delta ${ }^{\mathrm{MAX}}$ 
bioRxiv preprint doi: https://doi.org/10.1101/2022.03.07.483330; this version posted March 7, 2022. The copyright holder for this preprint (which was not certified by peer review) is the author/funder. All rights reserved. No reuse allowed without permission.

672 are referred to WT DLL4. ${ }^{*} \mathrm{P}<0.01, * * \mathrm{P}<0.001, * * * \mathrm{P}<0.001, * * * \mathrm{P}<0.0001$, ns: not significant 673 difference (Two-way ANOVA). T cell proliferation and IFN $\gamma$ secretion data were analyzed by 674 unpaired t tests.

675 


\section{$\underline{\text { Extended Data Material }}$}

6 An affinity-matured DLL4 ligand for broad-spectrum activation and 7 inhibition of Notch signaling

9 David Gonzalez-Perez ${ }^{1}$, Satyajit Das ${ }^{2}$, Elliot Medina ${ }^{1}$, Daniel Antfolk ${ }^{1}$, Emily D. Egan ${ }^{3}$, Stephen 10 C. Blacklow ${ }^{3}$, Paulo C. Rodriguez ${ }^{2}$, and Vincent C. Luca ${ }^{*}$.

$12{ }^{1}$ Department of Drug Discovery, Moffitt Cancer Center, Tampa, FL 33602, USA

132 Department of Immunology, Moffitt Cancer Center, Tampa, FL 33612, USA

$14{ }^{3}$ Department of Biological Chemistry and Molecular Pharmacology, Harvard Medical School, 15 Boston, Massachusetts 02115; Department of Cancer Biology, Dana Farber Cancer Institute, 16 Boston, Massachusetts 02115, USA

$17{ }^{*}$ Corresponding author: vince.luca@moffitt.org 


\section{MATERIAL AND METHODS}

\section{Reagents, media, and cell lines}

34 Mammalian cells were cultured at $37^{\circ} \mathrm{C}$, with a humidified atmosphere of $5 \% \mathrm{CO}_{2}$, washed with

35 Dulbecco's Phosphate Saline Buffer (DPBS, Corning), and detached with Trypsin-EDTA 0.25\%

36 (Gibco) for cell sub-culturing or cell-based assays. Notch reporter cell lines CHO-K1 hNotch1-

37 Gal4 were a gift from Dr. Michael Elowitz (California Institute of Technology) ${ }^{1}$. HEK293T, U2OS

38 and MCF-7 cells were cultured in high-glucose Dulbecco Eagle's Minimal Essential Medium

39 (DMEM, Cytiva) supplemented with 10\% Fetal Bovine Serum (FBS, peak serum) and 2\%

40 penicillin/streptomycin (Gibco). The MCF-7 breast cancer cell line was a gift from Dr. Eric Lau

41 (Moffitt Cancer Center). Hygromycin $100 \mu \mathrm{g} / \mathrm{ml}$ was added to U2OS reporter ${ }^{2}$ cell cultures to

42 maintain homogeneous populations of Notch-expressing cells. CHO-K1 hNotch1-Gal4 cells were

43 cultured in Minimum Essential Medium Eagle-alpha modification ( $\alpha$-MEM, Cytiva)

44 supplemented with 10\% Fetal Bovine Serum (FBS, peak serum), 2\% penicillin/streptomycin

45 (Gibco), $400 \mu \mathrm{g} / \mathrm{mL}$ of Zeocin (Alfa aesar) and $600 \mu \mathrm{g} / \mathrm{mL}$ of Geneticin (Gibco). K32 cells (K562

46 cell line expressing CD32) were a gift from Dr. Jose Conejo-Garcia (Moffitt Cancer Center), and

47 human $\mathrm{CD}^{+}$T cells were PBMCs (collected from LifeSouth Blood Bank, Gainesville, FL). Cells

48 used for $\mathrm{T}$ cell experiments were cultured at $37^{\circ} \mathrm{C}$, and $5 \% \mathrm{CO}_{2}$, in RPMI-1640 (Gibco)

49 supplemented with $2 \mathrm{mM}$ L-glutamine, $10 \mathrm{mM}$ HEPES, $150 \mathrm{U} / \mathrm{mL}$ streptomycin, $200 \mathrm{U} / \mathrm{mL}$

50 Penicillin, $20 \mathrm{mM} \beta$-mercaptoethanol and 10\% heat-inactivated FBS (GeminiBio). All cell lines

51 were tested negative for mycoplasma (ATCC), and stable cell lines were validated by sequencing

52 (Genewiz) after genomic DNA extraction (Invitrogen). Antibodies used in this study were: anti-

53 hNotch1 PE (Biolegend), anti-hNotch2 AF647 (Biolegend), anti-hNotch3 PE (Biolegend), anti-

54 hDLL4 PE (Biolegend), anti-hDLL1 PE (Biolegend), anti-hJagged1 AF647 (Biolegend), anti-

55 hJagged2 PE (Biolegend), and anti-HA tag AF488 (Cell Signaling Technologies), anti-hCD8a

56 Bv785 (Biolegend), anti-hIFN $\gamma$ APC (Biolegend), goat anti-mouse IgG (secondary antibody,

57 KPL), goat anti-rabbit IgG (secondary antibody, Vector laboratories), anti-cleaved Notch1

58 (Val1744, Cell Signaling Technology), anti-Notch2 (D76A6, Cell Signaling Technologies), anti-

$59 \beta$-Actin (Cell Signaling Technology and Sigma).

60 


\section{Protein expression and purification}

64 The minimum binding region of Delta-Like 4 (DLL4) fragments (N-EGF5) were cloned into the pAcGp67A vector containing an N-terminal gp67 signal peptide and C-terminal 8xHis-tag. Nonbiotinylated proteins were human wild-type DLL4 (N-EGF5, residues 27-400), DLL4.v1 (NEGF5), DLL4.v2 (N-EGF5), and DLL4 ${ }^{\mathrm{MAX}}$ (N-EGF5). Notch biotinylated fragments were cloned into pAcGp67A with a N-terminal gp67 signal peptide, a C-terminal 3C protease site followed by biotin-acceptor peptide tag (BAP-tag: GLNDIFEAQKIEWHE), and 6xHis-tag. In contrast, DLL4 biotinylated proteins contained BAP and His-tags, but lacked $3 \mathrm{C}$ site. Biotinylated proteins were human Notch1 (EGF8-12, residues 295-488), hNotch1 (EGF6-13, residues 237-549), hNotch2 (EGF6-13, residues 221-530), hNotch3 (EGF5-12, residues 197-505), hNotch4 (EGF7-12, residues 276-511), mouse Notch1 (EGF6-13, residues 221-526), WT DLL4 (N-EGF5, residues 27-400), and DLL4 ${ }^{\mathrm{MAX}}$ (N-EGF5, residues 27-400). All baculovirus-based constructs in this work were expressed for 48 hours by infecting HiFive Trichoplusia ni insect cell cultures (Expression Systems) at a density of $2 \times 10^{6}$ cells $/ \mathrm{mL}$ with infective baculovirus particles. Culture supernatants were harvested, and proteins purified by IMAC. Ni-NTA agarose resin (Qiagen) was washed with HEPES Buffered Saline (HBS: 20 mM HEPES pH 7.4, 150 mM sodium chloride; Buffer A) supplemented with $25 \mathrm{mM}$ imidazole (plus $1 \mathrm{mM}$ calcium chloride for Notch preps; Buffer A+C) and eluted with Buffer A (or Buffer $\mathrm{A}+\mathrm{C}$ for Notch samples) containing $250 \mathrm{mM}$ imidazole. Polishing was performed using a Superdex 200 Increase 10/300 GL column (GE) equilibrated in Buffer A for DLL4 proteins, or Superdex 75 Increase 10/300 GL column (GE) equilibrated in Buffer $\mathrm{A}+\mathrm{C}$ for Notch proteins. Biotinylated proteins were site-specifically modified in vitro at their C-terminal BAP-tag, using BirA ligase (in-house prepared) and polished as described above. Protein purity and integrity were analyzed by SDS-PAGE using TGX 12\% Pre-cast gels (BioRad). All proteins were flash frozen in liquid nitrogen and stored at $-80^{\circ} \mathrm{C}$ for later use.

\section{Affinity maturation of human DLL4 by directed evolution}

Starting point of evolution and structure-guided library creation

90 Wild-type human Delta-like 4 (N-EGF3, residues 27-322) was cloned into a modified version of

91 pCT302 an N-terminal fusion to an HA-epitope (YPYDVPDYA), c-Myc epitope

92 (EQKLISEEDL), and Aga2. A third binding site for DLL4:Notch interaction was designed on the

93 basis of the binding interface found in the crystal structure of rJagged1(N-EGF3):rNotch1(EGF8- 
94 12) complex ${ }^{3}$. The complex was analyzed with different computational tools (PDBePISA (EMBL95 EBI), Consurf server (Biosof), and PyMOL (Schrödinger)). Nine interface residues were selected

96 to create a structure-guided directed evolution library. Jagged1 homolog residues were identified

97 in DLL4 and targeted for mutagenesis. The mutant library was generated using assembly PCR

98 with the ten overlapping oligonucleotides below:

99 Lib1F:

100 CAGCCTATCTGTCTTTCGGGCTGTCATGAACAGAATGGCTACTGCAGCAAGC

101 Lib2R:

102 GCCAGCCTGGGCGGCAGAGGCACTCGGCCGGCTTGCTGCAGTAGCCATTC

103 Lib3F:

104 TCTGCCGCCCAGGCTGGCAGGGCCGGCTGTGTAACGAATGCATCCCC

105 Lib4R:

106 CCAGGGAGTGCTGCAGGTGCCGTGGCGACAGCCADDAWNGGGGATGCATTCGTTAC

107 ACAG

108 Lib5F:

109 CACCTGCAGCACTCCCTGGCAATGTMHTTGTGATGAGGGCTGGGGAG,

110 Lib6R:

111 GCAGTAGTTGAGATCTTGGTCACA

112 Lib7F:

113 GTGACCAAGATCTCAACTACTGCي VTCACCACTCCCCATGCAAGAATGGGGCAACG

114 TGC

115 Lib8R:

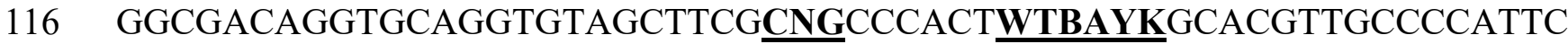

117 TTG

118 Lib9F:

119 CTACACCTGCACCTGTCGCCCGGGCTACACTGGTGTGGACTGTGAGGGATCCTACCC

120 ATAC

121 Lib10R:

122 AGATAAGCTTTTGTTCTCCACCAGCGTAATCTGGAACATCGTATGGGTAGGATCCCT

123 CAC). 
125 The primers contained specific degenerate codons (bold underlined) and 20bp overhangs 126 (Eurofins) to promote annealing in the PCR reaction. External primers were added to introduce a 127 40-50 bp recombination area between the mutant library region (EGF1-EGF3) and DLL4-pYAL 128 linearized vector. The mutant library was in vivo reassembled into yeast by electroporation (BTX 129 electroporator) with $10 \mu \mathrm{g}$ of linearized DLL4-pYAL vector and $50 \mu \mathrm{g}$ of the mutagenic library 130 (ratio 1:5 vector:library). Yeast transformants were recovered in SDCAA and DLL4 surface 131 displayed on S. cerevisiae EBY100 yeast as reported previously ${ }^{3,4}$. The theoretical library diversity 132 was 4x10E6, and we obtained an estimated library size of 8x10E6 variants (2-fold theoretical 133 diversity).

\section{Yeast display selections}

136 Before starting each selection round, induced yeast cultures were pelleted in logarithmic phase, 137 stained with anti-HA antibody conjugated to AF488 (Cell Signaling Technologies), and expression 138 of DLL4 on yeast surface analyzed by flow cytometry (BD Accuri C6 Plus). Selection rounds were 139 performed in two phases: (1) a negative selection to remove non-specific binders targeting 140 streptavidin and/or Alexa Fluor 647, and (2) a positive selection to rescue high-affinity variants

141 specific for Notch. Negative selection was performed by combining $100 \mathrm{nM}$ of streptavidin 142 conjugated to Alexa Fluor 647 (SA647) and $250 \mu \mathrm{L}$ anti-Alexa Fluor 647 magnetic beads 143 (Miltenyi) on ice for $10 \mathrm{~min}$. Next, the yeast library was washed once in selection buffer (HBS: 20 $144 \mathrm{mM}$ HEPES $\mathrm{pH}$ 7.4, $150 \mathrm{mM}$ sodium chloride, $1 \mathrm{mM}$ calcium chloride, 0.1\% Bovine Serum 145 Albumin (BSA) and $10 \mathrm{mM}$ maltose), and magnetic beads SA647-coated were added. The tube 146 was wrapped in foil and incubated at $4^{\circ} \mathrm{C}$ for $30 \mathrm{~min}$. Following this step, the yeast was washed 147 twice with selection buffer, resuspended, and flowed over a Magnetic-Activated cell sorting 148 (MACS) LS separation column (Miltenyi) pre-equilibrated in selection buffer. The flowthrough 149 was rescued, and positive selections were performed. For round zero (Naïve library), 8x10E7 cells 150 (10x library diversity) were used for selection against biotinylated Notch1 (EGF8-12) and Notch3 151 (EGF5-13), independently. Tetramers were pre-made on ice for $10 \mathrm{~min}$ by mixing $450 \mathrm{nM}$ of 152 biotinylated Notch1 (EGF8-12) or Notch3 (EGF5-12) with 100 nM of SA-647 (ratio 4.5:1). Next, $153100 \mathrm{nM}$ tetramers were added to $500 \mu \mathrm{L}$ of negatively selected yeast in selection buffer and 154 incubated for 2 hours at $4^{\circ} \mathrm{C}$. Yeast library stained with Notch tetramers was washed twice with $155500 \mu \mathrm{L}$ of selection buffer, and further incubated with $50 \mu \mathrm{L}$ of anti-AF647 magnetic beads for 30 
$\min$ at $4{ }^{\circ} \mathrm{C}$. After incubation, the yeast was washed twice with $500 \mu \mathrm{L}$ of selection buffer and highaffinity binders isolated by MACS columns. The isolated yeast cells were pelleted, recovered in 3 $\mathrm{mL}$ of SDCAA for 48 hours and induced in SGCAA for next round. For round 1, the same conditions were repeated like in round zero using Notch1 (EGF8-12) and Notch3 (EGF5-12) 100

$160 \mathrm{nM}$ tetramers for positive selections. Isolated populations were rescued in $3 \mathrm{~mL}$ SDCAA after 161 MACS sorting and induced in SGCAA. For round 2, 5x10E7 yeast cells were resuspended in 500 $162 \mu \mathrm{L}$ of selection buffer in the presence of $2 \mu \mathrm{M}$ monomer of Notch1 (EGF8-12) and Notch3 (EGF5163 12), independently. Tubes were wrapped in foil and incubated for 2 hours at $4^{\circ} \mathrm{C}$. Yeast samples 164 binding Notch were washed twice with $500 \mu \mathrm{L}$ of selection buffer, and incubated in $500 \mu \mathrm{L}$ of 165 selection buffer supplemented with $100 \mathrm{nM}$ of SA-647 for $30 \mathrm{~min}$ at $4^{\circ} \mathrm{C}$. DLL4 high-affinity 166 binders were isolated by MACS following the same approach described for rounds 1 and 2. Highaffinity binders for Notch1 and Notch3 were rescued in $3 \mathrm{~mL}$ of SDCAA for 48 hours and induced in SGCAA. For round 3, high-affinity populations were isolated by Fluorescent-Activated Cell Sorting (FACS, Sony Sorter SH800S). Only Notch3 (EGF5-12) was used since yeast-staining tests showed better population enrichment compared with Notch1 (EGF8-12). 1x10E8 yeast cells were resuspended into $500 \mu \mathrm{L}$ of selection buffer with pre-made $100 \mathrm{nM}$ tetramers of Notch3 (5-12)

172 and anti-HA488 antibody (double staining). The tube was wrapped in foil and incubated for 2 173 hours at $4^{\circ} \mathrm{C}$. Stained yeast was washed two times with $500 \mu \mathrm{L}$ of selection buffer and analyzed 174 by FACS. From the double-positive population (FIT-C+, APC+) showing expression of DLL4 175 (anti-HA488, FIT-C signal) and binding to Notch (SA-647, APC signal), a sorting gate was set at $176 \quad 1.8 \%$ of the maximum signal detected for APC channel and Notch binders isolated (Extended Data 177 Fig 1b). The sorted yeast population was washed two times with $5 \mathrm{~mL}$ of selection buffer, pelleted, 178 and recovered in SDCAA for 48 hours. Yeast cells were induced again in SGCAA for the last round of selection. For round 4, 5x10E7 cells were incubated with $0.2 \mu \mathrm{M}$ monomers of Notch3

180 (5-12) for 2 hours at $4^{\circ} \mathrm{C}$. Yeast cells were then washed twice with selection buffer, fluorescent181 labeled with SA-647 and high-affinity populations isolated by MACS following the protocol 182 described for previous rounds.

183 The isolated yeast populations obtained after round 4 were subjected to colony screen and plasmid 184 recovery by using Zymoprep kit (Zymo Research). The plasmids were transformed into E. coli 
R (5'-GGGATTTGCTCGCATATAGTTG) primers (Eurofins). Sequencing data analysis was performed using SnapGene software (Insightful Science).

\section{Generation of DLL4 variants}

190 We first generated a variant named DLL4.v1 by engrafting five affinity enhancing mutations into

191 human DLL4 scaffold that we previously reported for rat DLL4 affinity maturation (Site $1+$ Site

192 2; G28S, F107L, I143F, H194Y, and L206P) $)^{4}$. Next, the mutations found in this work to create a 193 third binding site on human DLL4 (Site 3; N257P, T271L, F280Y, S301R, Q305P) were combined 194 with Site $1+$ Site 2 mutations into one single DLL4 scaffold, creating an ultrapotent DLL4 protein, 195 named Delta ${ }^{\text {MAX }}$ (Site 1 + Site2; G28S, F107L, I143F, H194Y and L206P, and Site 3; N257P, 196 T271L, F280Y, S301R, Q305P).

\section{Thermal denaturation experiments by differential scanning fluorometry (DSF)}

199 WT DLL4 and Delta ${ }^{\mathrm{MAX}}$ (N-EGF5) were diluted at $5 \mu \mathrm{M}$ in $20 \mu \mathrm{L}$ of HBS buffer, and then combined with $10 \mu \mathrm{M}(5 \mathrm{x})$ SYPRO Orange (ThermoFisher Scientific) prepared in 100\% DMSO (final DMSO concentration was $0.1 \%$ ). This mix was equilibrated at room temperature for $30 \mathrm{~min}$. Proteins were analyzed in a 96-well microtiter plate using a StepOnePlus RT-PCR system (Applied Biosystems) applying a continuous heating gradient from 25 to $99^{\circ} \mathrm{C}$ with a step of $1 \%$ of temperature/min. Data was normalized and represented as the mean of three independent technical replicates. Similar Tm values were obtained for the replica measurements.

Determination of equilibrium dissociation constants $\left(K_{D}\right)$ by Surface Plasmon Resonance (SPR)

209 Equilibrium dissociation constants were determined by surface plasmon resonance using a Biacore

210 T200 instrument (GE Healthcare). Approximately 100 resonance units (RU) of biotinylated Notch 211 extracellular fragments (Notch1 (EGF6-13), Notch2 (EGF6-13), Notch3 (EGF5-12), Notch4 (7212 12), and mouse Notch1(EGF6-13)) were immobilized on individual flow cells at $5 \mu \mathrm{l} / \mathrm{min}$ using a 213 streptavidin-coated sensor chip (Series S Sensor chip SA, GE Healthcare). Three-fold serial 214 dilutions of recombinant DLL4 proteins (N-EGF5) starting at $50 \mu \mathrm{M}$ (WT DLL4), $18 \mu \mathrm{M}$ 215 (DLL4.v2), and $6 \mu \mathrm{M}$ (DLL4.v1 and Delta ${ }^{\mathrm{MAX}}$ ) were flowed over the chip at $25^{\circ} \mathrm{C}$ in SPR buffer 216 (20 mM HEPES pH 7.4, $150 \mathrm{mM} \mathrm{NaCl}, 1 \mathrm{mM}$ calcium chloride, 0.1\% BSA supplemented with 
$2170.005 \%$ surfactant P20). Association (“on-rate”) and dissociation ("off-rate") phases were 218 performed at $10 \mu \mathrm{l} / \mathrm{min}$ for 120 seconds and 300 seconds, respectively. The sensor chip was 219 regenerated after each injection with 30-second washes of 2.25M Magnesium Chloride containing $22025 \%$ Ethylene Glycol at $50 \mu \mathrm{L} / \mathrm{min}$. Data collection rate was performed at $10 \mathrm{~Hz}$, and curves were 221 reference-subtracted from a flow cell containing $100 \mathrm{RU}$ of a negative control non-related protein 222 (biotinylated mouse RNF43 PA domain). The maximum RU for each experiment was normalized 223 to a value of $100 \%$ and plotted as a function of concentration using Prism 9 software (GraphPad).

224 Steady-state binding and kinetic curves were fitted using the Biacore T200 evaluation software to 225 a 1:1 Langmuir model to determine the KD values.

\section{Generation of stable cell lines}

228 Ectodomains of WT DLL4 and Delta ${ }^{\mathrm{MAX}}$ (N-EGF8, residues 27-518) were cloned into an 229 intermediary vector containing WT DLL4 signal peptide, juxtamembrane, transmembrane, and 230 intracellular domain, fused to C-terminal Myc (EQKLISEEDL) and Flag (DYKDDDDK) tags. 231 This vector was generated after cloning of a synthetic DNA fragment (Eurofins) into pLenti-C232 Myc-DDK-IRES-Puro vector (Invitrogen). For stable cell lines expressing DLL1 (residues 1-723), 233 Jagged1 (residues 1-1218), and Jagged2 (residues 1-1238), their CDS was cloned into pLenti-C234 Myc-DDK-IRES-Puro vector fused to c-Myc and Flag C-termini tags. HEK-293T mammalian 235 cells were used for lentiviral particle production and transductions (kindly donated by Dr. Eric 236 Lau, Moffitt Cancer Center). Briefly, transfections were carried out with packaging vectors VSV$237 \mathrm{G}$ and d8.9 (kindly donated by Dr. Eric Lau, Moffitt Cancer Center) in the presence of 238 Polyethyleneimine (PEI) at ratio 4:1 (DNA:PEI). Lentiviral particles were harvested after 48-72 239 hours and used to transduce HEK293T cells using $1 \mu \mathrm{g} / \mathrm{mL}$ polybrene (Millipore). Antibiotic 240 selection was performed using Puromycin at $5 \mu \mathrm{g} / \mathrm{mL}$ (Gibco) for 10-15 days. Puromycin resistant 241 cells expressing WT DLL4 and Delta ${ }^{\mathrm{MAX}}$ were detached with trypsin-free dissociation buffer 242 (Gibco), stained in DPBS + 0.5\% BSA with anti-hDLL4 PE antibody for 1 hour at $4{ }^{\circ} \mathrm{C}$, and then 243 sorted by FACS (Sony sorter SH800S). Sorted cells (mean fluorescence intensity around 1x10E5$2443 \times 10 \mathrm{E} 5)$ were washed with DPBS and recovered in DMEM $+10 \% \mathrm{FBS}$ at $37^{\circ} \mathrm{C}$ and $5 \% \mathrm{CO}_{2}$ for 2 245 weeks. Expression of WT DLL4 and Delta ${ }^{\mathrm{MAX}}$ on HEK293T surface was confirmed by flow 246 cytometry (BD Accuri C6 plus) staining the cell lines with anti-hDLL4 PE in DPBS $+0.5 \%$ BSA 247 (Extended Data Fig 7b). DLL1, Jagged1, and Jagged2 cell lines were stained with specific 
antibodies (anti-hDLL1 PE, anti-hJagged1 AF647, and anti-hJagged2 PE) for 1 hour at $4^{\circ} \mathrm{C}$ and analyzed by flow cytometry (BD Accuri C6 plus); however, these cell lines did not require further sorting steps (Extended Data Fig. 10).

252 K32 cells (K562 cells expressing human CD32) were transduced with lentivirus expressing WT DLL4 or Delta ${ }^{\mathrm{MAX}}$ by spin-infection using $8 \mu \mathrm{g} / \mathrm{mL}$ polybrene (Millipore). Seventy-two hours after transduction, cells were analyzed for DLL4 expression and further cultured in RPMI $+10 \%$ FBS containing $1 \mu \mathrm{g} / \mathrm{ml}$ of puromycin (Gibco) at $37{ }^{\circ} \mathrm{C}$ and $5 \% \mathrm{CO}_{2}$. DLL4 expression was monitored by flow cytometry (CytoFLEX II, Beckman Coulter) using anti-hDLL4 PE (Biolegend) until the culture reached $\sim 100 \%$ of positive population on PE signal.

\section{Notch activation assays with ligands non-specifically adsorbed to plates}

260 On day one, 2-fold serial dilutions of non-biotinylated DLL4 recombinant proteins (N-EGF5) were prepared in $100 \mu \mathrm{L}$ of DPBS and used to coat 96-well plates (Coastar) for 1 hour at $37^{\circ} \mathrm{C}$. WT DLL4 started at $50 \mu \mathrm{M}$ and Delta ${ }^{\mathrm{MAX}}$ at $204 \mathrm{nM}$. Then, the wells were washed three times with $200 \mu \mathrm{L}$ of DPBS to remove unbound proteins. Next, CHO-K1 Notch1-Gal4 cells in culture were detached with trypsin-EDTA $0.25 \%$ (Gibco) and manually counted. Appropriate dilutions were prepared in $\alpha$-MEM media to ensure 30,000 CHO-K1 cells per well in $200 \mu \mathrm{L}$. Ligand-coated plates and reporter cell lines were incubated for 24 hours at $37^{\circ} \mathrm{C}$ and $5 \% \mathrm{CO}_{2}$. On day two, CHOK1 Notch1-Gal4 cells were washed with $200 \mu \mathrm{L}$ DPBS, detached with $30 \mu \mathrm{L}$ of trypsin-EDTA $0.25 \%$, and quenched with $200 \mu \mathrm{L}$ of $\alpha$-MEM media. Finally, cells were resuspended and H2BmCitrine signal was measured by flow cytometry (BD Accuri C6 plus). CHO-K1 Notch1-Gal4 and ligand-lacking wells were used as a negative control. The measurements represent the mean fluorescent intensity as percentage of Notch activation \pm S.D. of three biological replicates. Notch activation was reference-subtracted from a well coated with $0.5 \%$ BSA containing CHO-K1 cells.

273 Gating strategies for this experiment are detailed in Extended Data Fig. 7a.

\section{Notch activation with ligand-oriented coupling on streptavidin plates}

276 For CHO K1 reporters, on day one, tissue culture 96-well plates (Coastar) were pre-treated with $277100 \mu \mathrm{L}$ of streptavidin at $10 \mu \mathrm{g} / \mathrm{mL}$ (VWR) for 1 hour at $37^{\circ} \mathrm{C}$, washed three times with $200 \mu \mathrm{L}$ 
removed by washing three times with $200 \mu \mathrm{L}$ DPBS (Corning), and $100 \mu \mathrm{L}$ of DPBS containing

280 biotinylated DLL4 proteins were coupled to streptavidin for 1 hour at $37^{\circ} \mathrm{C}$ (2-fold serial dilutions

281 starting at $50 \mathrm{nM}$ ). Non-coupled DLL4 proteins were removed by washing three times with 200

$282 \mu \mathrm{L}$ DPBS. Next, Notch reporter cells in culture were detached with trypsin-EDTA 0.25\% (Gibco),

283 and 30,000 cells in $\alpha$-MEM media $(200 \mu \mathrm{L})$ were added into individual wells. Assay plates were

284 incubated for 24 hours at $37^{\circ} \mathrm{C}$, and 5\% CO . On day two, CHO-K1 Notch1-Gal4 cells were

285 washed with $200 \mu \mathrm{L}$ DPBS, detached with $30 \mu \mathrm{L}$ of trypsin-EDTA $0.25 \%$, and $200 \mu \mathrm{L}$ of $\alpha$-MEM

286 media added. Cells were resuspended and Notch activation was measured as a function of

287 fluorescence by flow cytometry (BD Accuri C6 plus). CHO-K1 Notch1-Gal4 cells and lacking-

288 ligand wells were used as a negative control. The measurements represent the mean fluorescent

289 intensity as percentage of Notch activation \pm S.D. of three biological replicates. Notch activation

290 was reference-subtracted from a well coated with a non-related control protein (PA domain of

291 RNF128).

293 For U2OS reporter cells, approximately 5x10E5 cells were plated into 6-well plates (Falcon) and

294 let to adhere overnight at $37^{\circ} \mathrm{C}, 5 \% \mathrm{CO}_{2}$ in DMEM media (Cytiva). On day two, media was 295 replaced with serum-free DMEM and cells transfected with luciferase vectors. Cationic lipoplexes 296 were generated for $15 \mathrm{~min}$ at room temperature by mixing $10 \mu \mathrm{L}$ of Lipofectamine 2000 297 (Invitrogen), 2.5 $\mathrm{g}$ of Gal4-firefly luciferase reporter, and $50 \mathrm{ng}$ of pRL-TK Renilla luciferase 298 vector as internal control (ratio 1:5, Lipo:DNA). On day three, streptavidin plates were coupled 299 with DLL4 ligands like described above using 10-fold serial dilutions starting at $50 \mathrm{nM}$, and 300 transfected U2OS cells were detached with Trypsin-EDTA, washed with DPBS, and counted in 301 the microscope. Twenty-thousand reporter cells were added to wells using $200 \mu \mathrm{L}$ of DMEM 302 media containing $2 \mu \mathrm{g} / \mathrm{mL}$ of doxycycline (Sigma) to induce Notch-Gal4 expression. Next, 303 signaling plates were incubated for 24 hours at $37^{\circ} \mathrm{C}$ and $5 \% \mathrm{CO}_{2}$. On day four, luciferase firefly 304 and Renilla signals were determined with Dual-Glo luciferase assay system (Promega) using a 305 GloMax luminometer (Promega). A luminescence ratio firefly:Renilla was determined for every 306 well and normalized to the signal of a non-related control protein (PA domain of RNF128). Notch 307 activation is represented as the mean fold-change over the control \pm S.D. of three independent 308 biological replicates. 


\section{Kinetics for Notch activation assay}

96-well tissue culture plates (Coastar) were pre-coated using ligand-oriented coupling as described above with $50 \mathrm{nM}$ of DLL4 biotinylated variants. CHO-K1 reporter cells were detached with trypsin-EDTA, washed with DPBS, and $200 \mu \mathrm{L}$ of $\alpha$-MEM media containing 30,000 cells were added into wells. Notch activation was analyzed as end-points for 0, 4, 8, 12, 16, 20, and 24-hour measurements. Desired time point wells containing CHO-K1 reporter cells were washed once with $200 \mu \mathrm{L}$ DPBS, detached with trypsin-EDTA, and quenched with $200 \mu \mathrm{L}$ of $\alpha$-MEM media. Next, reporter cells were resuspended and analyzed by flow cytometry reading the fluorescence of H2BmCitrine (BD Accuri C6 Plus). Every time point represents the mean fluorescence intensity of Notch activation as percentage \pm S.D. of three independent biological replicates. Notch activation was reference-subtracted from a well containing streptavidin, BSA and CHO-K1 cells.

\section{Notch activation in co-culture format with HEK293T}

On day one, U2OS reporter cells (signal receiver cells) were detached with trypsin-EDTA, washed with DPBS, and 5x10E5 cells were added to 6-well plates (Falcon) for transfection with luciferase reporter vectors. On day two, U2OS cells were transfected as described above. On day three, signal receiver cells were detached with trypsin-EDTA, and $100 \mu \mathrm{L}$ of DMEM media containing 20,000 cells were added to tissue culture 96-well plates (Coastar). In parallel, HEK293T-expressing WT DLL4 or Delta ${ }^{\mathrm{MAX}}$ were detached with trypsin-EDTA, counted manually, and dilutions prepared such that $100 \mu \mathrm{L}$ of DMEM containing 20,000 signal sender cells were added over signal receiver cells (cell ratio 1:1). Doxycycline (Sigma) at $2 \mu \mathrm{g} / \mathrm{mL}$ was used to induce Notch-Gal4 expression.

331 On day four, luciferase firefly and Renilla signals were determined with Dual-Glo luciferase assay 332 system (Promega) using a GloMax luminometer (Promega). A luminescence ratio firefly:Renilla 333 was determined for every well and normalized to the signal of a well containing U2OS reporter 334 cells and HEK293T non-expressing DLL4 ligands. Notch activation is represented as the mean fold-change over the control \pm S.D. of three independent biological replicates.

337 Notch signaling assay using multiple ligand-presentation formats

338 Notch activation was assayed in different formats (Extended Data Fig. 8a) including (1) non339 specific orientation (100 nM of Delta ${ }^{\mathrm{MAX}}$ on well, Fig. 3a), (2) ligand-oriented coupling (10 nM 340 of Delta ${ }^{\mathrm{MAX}}$ on well, Fig. 3b), (3) magnetic beads coated with biotinylated Delta ${ }^{\mathrm{MAX}}$ (ratio 1:20 
341 reporter cells:beads, optimization of ratio is detailed in Extended Data Fig. 8d), (4) HEK293T342 expressing Delta ${ }^{\mathrm{MAX}}$ (ratio 1:1, signal receivers:senders, optimization of ratio can be seen in 343 Extended Data Fig. 8c), and (5) yeast-expressing Delta ${ }^{\mathrm{MAX}}$ (ratio 1:10, reporter:yeast, 344 optimization of ratio is detailed in Extended Data Fig. 8b). On day one, U2OS hNotch1-Gal4 cells 345 were detached with trypsin-EDTA, counted in the microscope, and 5x10E5 cells/well transferred 346 to 6-well tissue culture plates (Falcon). On day two, U2OS cells were transfected as described 347 above. On day three, the different activation assays were set up at the same time. For ligand 348 oriented-coupling, streptavidin plates were coated with $10 \mathrm{nM}$ of Delta ${ }^{\mathrm{MAX}}$ as described above. 349 For non-specific adsorption, non-biotinylated Delta ${ }^{\mathrm{MAX}}$ at $100 \mathrm{nM}$ was coated as described 350 previously. For activation using streptavidin beads coated with biotinylated Delta ${ }^{\mathrm{MAX}}, 200 \mathrm{mg}$ of 351 streptavidin beads (streptavidin MagneSphere paramagnetic particles, Promega) were washed 3 352 times with $200 \mu \mathrm{L}$ of DPBS $+0.1 \%$ BSA using a magnet (Miltenyi), and combined with $20 \mu \mathrm{g}$ of 353 biotinylated Delta ${ }^{\mathrm{MAX}}$ for 1.5 hours at $4^{\circ} \mathrm{C}$. Next, unbound Delta ${ }^{\mathrm{MAX}}$ was washed out three times 354 with $200 \mu \mathrm{L}$ of DPBS $+0.1 \%$ BSA, and $100 \mu \mathrm{L}$ of complexed beads (pre-diluted 1/25 in DPBS) 355 were added to wells. For co-culture activation with HEK293T-expressing Delta ${ }^{\mathrm{MAX}}$, cells were 356 detached with trypsin-EDTA, quenched with DMEM media, washed with DPBS, and $100 \mu \mathrm{L}$ 357 containing 20,000 cells in DMEM were added to wells. For activation with yeast cells expressing 358 Delta $^{\mathrm{MAX}}$ on surface, pYAL vector encoding for Delta ${ }^{\mathrm{MAX}}$ (N-EGF5) was electroporated 359 previously into yeast cells, and DLL4 expressed in SGCAA as previously described. Delta ${ }^{\text {MAX }}$ 360 expression was induced 48 hours before Notch activation assay set up. One hundred microliters of 361 yeast cells $(2 \times 10 E 6$ cells $/ \mathrm{mL})$ in serum-free DMEM were added to the assay plate. Once all the 362 wells contained the desired activation assays, U2OS reporter cells were detached with trypsin363 EDTA, and 20,000 cells in $100 \mu \mathrm{L}$ of DMEM were added per well. All the conditions were 364 supplemented with $2 \mu \mathrm{g} / \mathrm{mL}$ of doxycycline to induce Notch1-Gal4 expression. The assay plate 365 was incubated for 24 hours at $37^{\circ} \mathrm{C}$ and $5 \% \mathrm{CO}_{2}$. On day four, media was aspirated off and 366 Luciferase Dual-Glo kit (Promega) used to detect firefly and Renilla signals in a luminometer 367 (GloMax, Promega). Negative controls were BSA 0.5\% for non-specific absorption, streptavidin 368 and BSA $2 \%$ for ligand oriented-coupling, uncoated streptavidin beads for activation with 369 magnetic beads, HEK293T non-expressing DLL4 for co-culture activation, and yeast expressing 370 PDL-1 ECD for co-culture with yeast. The expression of Delta ${ }^{\mathrm{MAX}}$ in HEK293T and yeast cells 371 was confirmed by flow cytometry (BD Accuri C6 plus) following staining for 1 hour at $4^{\circ} \mathrm{C}$ with 
anti-hDLL4 PE antibody using DPBS + 0.5\% BSA, and selection buffer, respectively (Extended

373 Data Fig 8e). Notch activation is represented as the mean fold-change over the corresponding 374 control \pm S.D. of three independent biological replicates.

\section{Notch inhibition assays with soluble antagonists and WT DLL4 coated plates}

377 For CHO-K1 reporter cells, on day one, biotinylated WT DLL4 was pre-coated in 96-well plates at $50 \mathrm{nM}$ following the same protocol described above. Next, CHO cells were detached with trypsin-EDTA, washed, and diluted at 200,000 cells/mL in $\alpha$-MEM media. Ten-fold serial dilutions of non-biotinylated DLL4 ligands (N-EGF5) were prepared in DPBS starting at $300 \mathrm{nM}$. Next, soluble inhibitors were mixed in ratio 1:1 with CHO-K1 200,000 cells/mL stocks for $15 \mathrm{~min}$ at room temperature to pre-block Notch receptors. Two hundred microliters of inhibition reactions containing 20,000 reporter cells were added to WT DLL4-coated wells, and plates incubated for 24 hours at $37^{\circ} \mathrm{C}$ and $5 \% \mathrm{CO}_{2}$. When DAPT (gamma-secretase inhibitor, Sigma) and BB-94 (ADAM metalloprotease inhibitor, Sigma) were used, stock solutions were prepared at $100 \mu \mathrm{M}$ in 8\% DMSO. Appropriate DMSO controls were included to test Notch activation and cell viability. Final DMSO concentration in the experiment was kept below 1\%. For comparisons of Delta $\mathrm{MAX}$ with DAPT and BB-94, soluble inhibitors were serially diluted in DPBS using 9-folds, incubated with $\mathrm{CHO}$ reporter cells, and added to wells as described above. On day two, assay plates were washed with $200 \mu \mathrm{L}$ of DPBS, CHO cells detached with trypsin-EDTA, quenched with $200 \mu \mathrm{L}$ of $\alpha-M E M$ media, and Notch activation measured by flow cytometry (BD Accuri C6 Plus). The data represents the mean fluorescent intensity as percentage of Notch activation, relative to Notch activation in the absence of inhibitors \pm S.D. of three independent biological replicates.

395 For U2OS cells, on day one, 5x10E5 cells were plated in 6-well tissue culture plates. On day two, U2OS cells were transfected as described above. On day three, 96-well plates (Coastar) were coated with streptavidin and biotinylated WT DLL4 at $50 \mathrm{nM}$ as described above. Next, U2OS 398 reporter cells were detached with trypsin-EDTA and diluted at 200,000 cells/mL in DMEM media. 399 Ten-fold serial dilutions of soluble DLL4 variants starting at 3,000 nM were prepared in DPBS 400 and used to pre-block reports cells following the same protocol described above. Two-hundred 401 microliters of inhibition reactions were added to 96-well plates pre-coated with biotinylated WT 
403 fourth, luciferase signals were measured with Dual-Glo luciferase assay system (Promega) using 404 a GloMax luminometer (Promega). Doxycycline at $2 \mu \mathrm{g} / \mathrm{mL}$ was maintained to ensure binding of 405 soluble inhibitors to Notch-pan receptor. A ratio between firefly and renilla luciferase was 406 calculated, and Notch activation normalized to wells where inhibitors were non-added. The data 407 represent fold-change of Notch activation as percentage, relative to Notch activation in the absence 408 of inhibitors \pm S.D. of three independent biological replicates.

\section{Notch inhibition assay in co-culture format}

411 For inhibition of Notch ligand-expressing cell lines, on day one, U2OS Notch3 reporter cells 412 (signal receivers) were detached with trypsin-EDTA, washed with DPBS, and added to 6-well 413 plates. On day two, U2OS cells were transfected as described above. On day three, signal receiver 414 cells were detached with trypsin-EDTA, and dilutions of 400,000 cells/mL were prepared in 415 DMEM. On the one hand, soluble DLL4 variants were prepared at $6 \mu \mathrm{M}$ in DPBS, combined in 416 1:1 dilution with signal receiver cells, and incubated for $15 \mathrm{~min}$ at RT. On the other hand, 417 HEK293T-expressing WT DLL4, DLL1, JAG1, JAG2 were detached with trypsin-EDTA, washed 418 with DPBS and 200,000 cells/mL stocks prepared in DMEM. One hundred microliters of both 419 signal senders and pre-blocked signal receivers were combined in cell ratio 1:1 and incubated for 42024 hours at $37^{\circ} \mathrm{C}$, and $5 \% \mathrm{CO}_{2}$. On day four, luciferase firefly and Renilla signals were determined 421 with Dual-Glo luciferase assay system (Promega) using a GloMax luminometer (Promega). 422 Doxycycline at $2 \mu \mathrm{g} / \mathrm{mL}$ was kept for all the conditions. A luminescence ratio firefly:Renilla was 423 determined for every well. Notch activation of each Notch3:ligand pair was normalized to the 424 signal of Notch3 reporters in the presence of HEK293T lacking ligands. Each Notch signaling pair 425 was set at $100 \%$ activation and soluble DLL4 treatments were relative to its corresponding pair. 426 The data represent fold-change of Notch activation as percentage, relative to Notch activation in 427 the absence of inhibitors \pm S.D. of three independent biological replicates.

429 For inhibition of CHO-K1 cells with DAPT and Delta ${ }^{\mathrm{MAX}}$, the experiment was set-up following 430 the protocol described above with the exception that CHO-K1 reporters were incubated with 431 HEK293T-expressing WT DLL4 for Notch activation. Three-fold serial dilutions of soluble 432 Delta $^{\mathrm{MAX}}$ and DAPT were prepared in DPBS starting at $3 \mu \mathrm{M}$. For DAPT, a stock solution of 100 $433 \mu \mathrm{M}$ in 8\% DMSO was prepared before serial dilutions in DPBS. Appropriate DMSO controls were 
434 also assayed for cytotoxicity. Final DMSO concentration in the wells was maintained below $1 \%$.

435 Thirty thousand signal senders and pre-blocked signal receivers (100 $\mu \mathrm{L}$ each) were incubated for

43624 hours at $37^{\circ} \mathrm{C}$ and $5 \% \mathrm{CO}_{2}$ before analysis by flow cytometry. Maximum Notch activation

$437(100 \%)$ was set in the absence of soluble inhibitors and normalized to wells containing CHO-K1

438 cells and HEK293T lacking expression of WT DLL4. Mean fluorescence intensity is represented

439 as percentage relative to Notch activation in the absence of inhibitors \pm S.D. of three independent

440 biological replicates.

\section{Co-culture assays for $\mathbf{T}$-cell activation and proliferation}

443 Functional assays for human $\mathrm{CD}^{+} \mathrm{T}$ cells were performed in co-culture with K562 cells 444 expressing human CD32 (K32 cells) generated as described before ${ }^{5}$. Briefly, cells were $\gamma$ 445 irradiated (100Gy) and washed twice with PBS before being loaded with OKT3 (0.0125$4460.5 \mu \mathrm{g} / \mathrm{mL}$, BioXcell) antibody at RT for $10 \mathrm{~min}$. Human $\mathrm{CD}^{+} \mathrm{T}$ cells were negatively isolated 447 from PBMC and labeled with $5 \mu \mathrm{M}$ of Cell Trace Violet (Invitrogen) according to manufacturer's 448 specifications. Next, $\mathrm{CD}^{+} \mathrm{T}$ cells were co-cultured with $\mathrm{K} 32$ cells at ratio 1:10 (T cell: K32). The

449 frequency of proliferating $\mathrm{T}$ cells was determined after 96 hours by flow cytometry using a 450 CytoFLEX II (Beckman Coulter). Zombie Green (Biolegend) was used for life/dead cell staining 451 to discard dead cells from the analysis. A set of samples were additionally incubated with Golgi 452 stop (BD) at $0.5 \mu \mathrm{L} /$ well for 5 hours and washed with PBS for detection of intracellular IFN $\gamma$ using 453 anti-hIFN $\gamma$ APC antibody (Biolegend). The frequency of IFN $\gamma$ positive cells was analyzed by flow 454 cytometry (CytoFLEX II, Beckman Coulter). Dead cells were excluded from the analysis by 455 staining with Zombie green (Biolegend). The data represents the mean percentage of proliferating $456 \mathrm{~T}$ cells or IFN $\gamma^{+} \mathrm{T}$ cells \pm S.E.M of four independent biological replicates.

\section{Quantitative Real-time PCR}

459 Human $\mathrm{CD}^{+} \mathrm{T}$ cells were co-cultured with K32-DLL4 cells for 96 hours and positively enriched 460 by MACS column (Invitrogen Kit). Total RNA was isolated from $\mathrm{CD}^{+} \mathrm{T}$ cells using TRIzol (Life 461 Technologies). Reverse transcription reaction was performed using Verso cDNA Synthesis Kit 462 (Thermo Scientific). Quantitative PCR reactions were prepared by using Bio-Rad SYBR green 463 master mix and performed on an Applied Biosystems thermocycler (7900 HT). Relative expression 464 was calculated using the $\Delta \Delta \mathrm{Ct}$ method and normalized to $\beta 2 \mathrm{M}$ levels. The data represents the mean 
465 quantity of mRNA (in-fold) for different $T$ cell markers \pm S.E.M of four independent biological

466 replicates. Pair primers used were:

467 Human $\beta 2-$ Microglobulin

468 Forward: 5'-ATGAGTATGCCTGCCGTGTGA

469 Reverse: 5'-GGCATCTTCAAACCTCCATG

470 Human IFN $\gamma$

471 Forward: 5'-GACCAGAGCATCCAAAAGAG

472 Reverse: 5'-GGACATTCAAGTCAGTTACCGAATA

473 Human Granzyme B

474 Forward: 5'-CCCTGGGAAAACACTCACACA

475 Reverse: 5'-GCACAACTCAATGGTACTGTCG)

476 Human Hes-4

477 Forward: 5'-ACGGTCATCTCCAGGATGT

478 Reverse: 5'-CGAGCGCGTATTAACGAGAG)

\section{Immunoblot Analysis}

481 For detection of Notch activation in $\mathrm{CD}^{+} \mathrm{T}$ cells, following co-culture with $\mathrm{K} 32$ cell lines, $\mathrm{CD} 8^{+}$ $482 \mathrm{~T}$ cells were positively enriched by Magnisort Human $\mathrm{CD}^{+} \mathrm{T}$ cell positive selection Kit 483 (Invitrogen). Nuclear fraction was isolated using NE-PER Nuclear and Cytoplasmic extraction kit 484 (ThermoFisher). Equal protein amounts of nuclear cell lysates were run on 4-20\% gradient Tris485 glycine gels (Novex-Invitrogen), transferred to PVDF membranes using an iBlot Gel Transfer 486 Device (ThermoFisher), and immunoblotted with antibodies anti-cleaved Notch1 (Val1744 rabbit $487 \mathrm{mAb}$, Cell Signaling Technology, 1:1,000), anti-Notch2 (D76A6 rabbit mAb, Cell Signaling 488 Technology, 1:1,000), and anti- $\beta$ Actin (mouse mAb, Sigma, 1:20,000). Secondary antibodies anti489 IgG (anti-rabbit or anti-mouse, GE Healthcare, 1:5,000) conjugated to horseradish peroxidase 490 (HRP) were used for detection of proteins using ECL Western Blot Substrate Reagent (Thermo 491 Fisher Scientific). Images were acquired using a Chemidoc Imaging System and analyzed with 492 Image-Lab software (Bio-Rad).

494 For Notch activation in U2OS and MCF-7 cells, WT DLL4 and Delta ${ }^{\text {MAX }}$ biotinylated ligands 495 were immobilized at $50 \mathrm{nM}$ in 6-well streptavidin-coated plates following a scaled up protocol of 
496 the one described before. After 24 hours of stimulation, cells were lysed in Laemmli serum buffer 497 (40\% glycerol, 4\% SDS, $250 \mathrm{mM}$ Tris-HCl, $0.02 \%$ bromophenol blue, and $20 \% \quad \beta-$ 498 mercaptoethanol). Cell lysates were heated at $100^{\circ} \mathrm{C}$ for $5 \mathrm{~min}$, centrifuged at $21,000 \mathrm{xg}$ for $10 \mathrm{~min}$ 499 at $4^{\circ} \mathrm{C}$, and supernatants stored at $-20^{\circ} \mathrm{C}$. Proteins were separated by SDS-PAGE (12\% Mini500 PROTEAN TGX Precast Protein Gels, Bio-Rad) and transferred to PVDF membranes using an 501 iBlot2 Gel Transfer Device (Thermo Fisher Scientific). The membranes were blocked in 3\% BSA $502+0.1 \%$ TBS-Tween. Primary antibodies were anti-cleaved Notch1 (Val1744 rabbit mAb, Cell 503 Signaling Technology, 1:1,000), and $\beta$-Actin (rabbit pAb, Cell Signaling Technology, 1:1,000).

504 Secondary antibody anti-Rabbit IgG conjugated to HRP (Goat mAb, Vector Laboratories, 1:8,000) 505 was used for detection of proteins using SuperSignal West Pico PLUS Chemiluminescent 506 Substrate (Thermo Fisher Scientific). Images were acquired using an Amersham Imager 600 (GE 507 Healthcare), and contrast adjusted with a curves layer.

\section{References}

510 1. Sprinzak, D. et al. Cis-interactions between Notch and Delta generate mutually exclusive 511 signalling states. Nature 465, 86-90 (2010).

512 2. Malecki, M. J. et al. Leukemia-Associated Mutations within the NOTCH1 Heterodimerization 513 Domain Fall into at Least Two Distinct Mechanistic Classes. Mol Cell Biol 26, 4642-4651 (2006).

514 3. Luca, V. C. et al. Notch-Jagged complex structure implicates a catch bond in tuning ligand 515 sensitivity. Science 355, 1320-1324 (2017).

516 4. Luca, V. C. et al. Structural basis for Notch1 engagement of Delta-like 4. Science 347, 847-853 517 (2015).

518 5. Maus, M. V. et al. Ex vivo expansion of polyclonal and antigen-specific cytotoxic $\mathrm{T}$ 519 lymphocytes by artificial APCs expressing ligands for the T-cell receptor, CD28 and 4-1BB. Nat Biotechnol 20, 143-148 (2002). 
EXTENDED DATA FIGURES

528

a

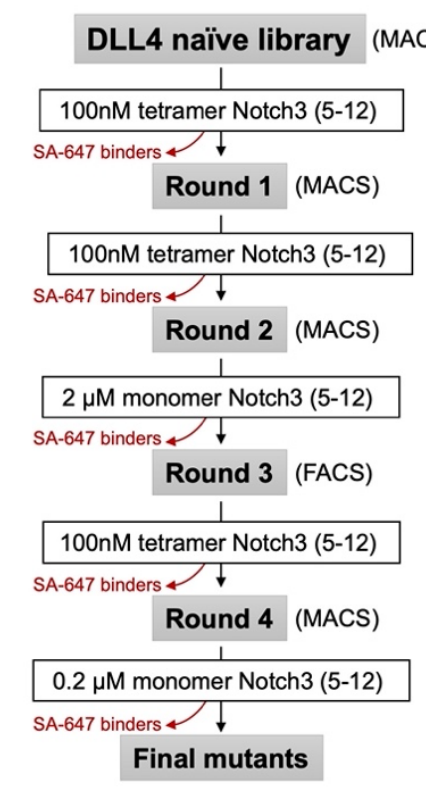

b
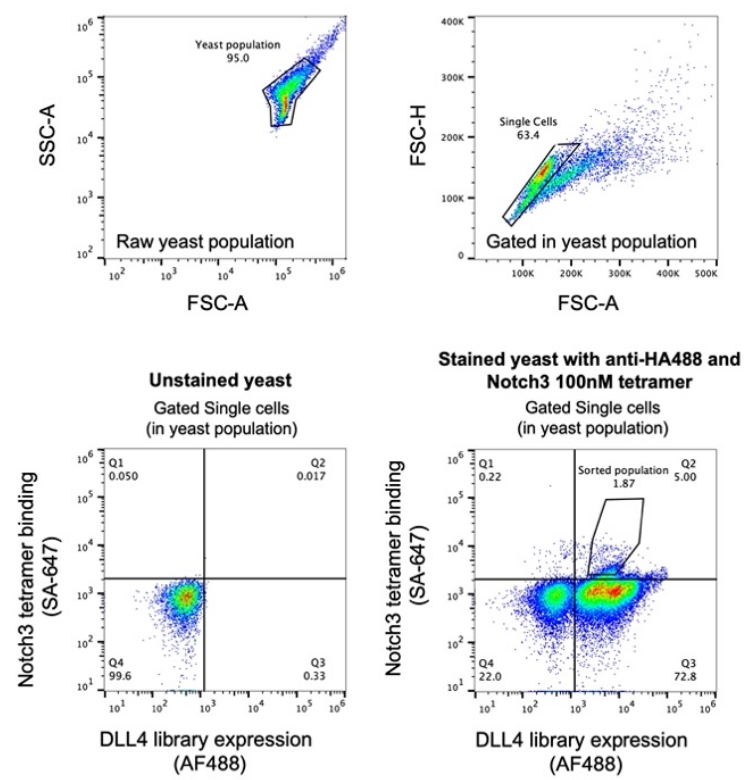

530 Extended Data Fig 1. Selection strategy for isolation of high-affinity DLL4 populations. (A)

531 Flow chart depicting the selection strategy used to isolate high-affinity DLL4 variants. Red arrows

532 and text indicate negative selections. (B) Gating strategy for sorting high-affinity binders to

533 Notch3. 
a

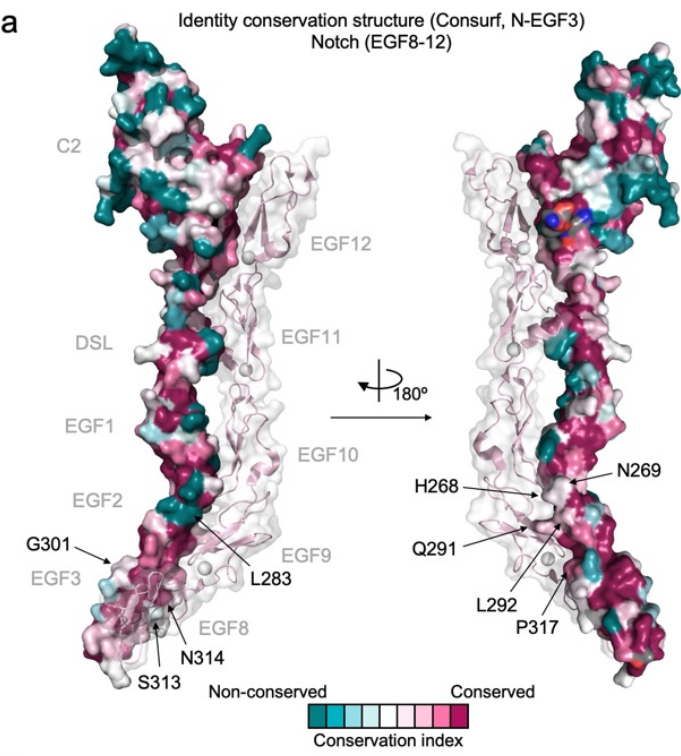

b

\begin{tabular}{|c|cccc|}
\multicolumn{4}{c}{} & \multicolumn{3}{c|}{ Identity matrix (\%) } \\
\cline { 2 - 5 } \multicolumn{1}{c|}{} & JAG1 & JAG2 & DLL4 & DLL1 \\
\hline JAG1 & 100.0 & 62.1 & 44.6 & 44.7 \\
JAG2 & 62.1 & 100.0 & 44.2 & 46.6 \\
hDLL4 & 44.6 & 44.2 & 100.0 & 59.7 \\
hDLL1 & 44.7 & 46.6 & 59.7 & 100.0 \\
\hline
\end{tabular}

c

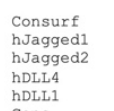

hDLL1

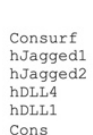

hDLL1

Consurf

hJagged1

hJagged
hDLL4

hDLL:

Consurf

Consurf
hJagged1
hJagged2
hDLL4

hDLL4

Cons

Consurf
hJagged1
hJagged2
hDLL4
hDLL1
Cons

Consurf
hJagged1
hJagged2
hDLL4
hDLL1
Cons
Consurf alignment of Notch ligands

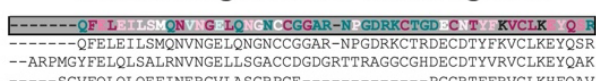

----SGVFQLLLQEFINERGVLASGRPCE-------------PGCRTFRRVCLKHEQA CQVWSSGVFELKLQEFVNKKGLLGNRNCCRGGAGPP------PCACRTFFRVCLKHYQAS

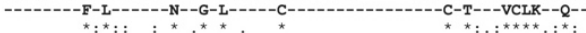

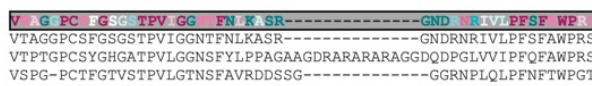
VSPEPPCIYGSAVTPVGVDSFSLPDGGGAD----------SAFSNPIRFPFGFTWPG

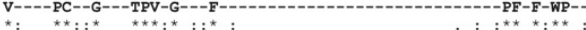

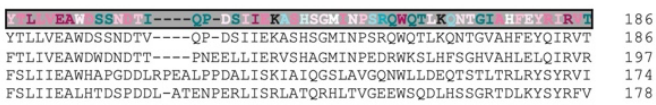

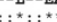
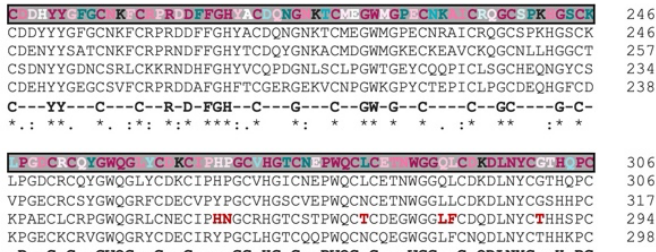
KPAECLCRPGWQGRLCNECIPHNGCRHGTCSTPWQCTCDEGWGGLFCDQDLNYCTHHSPC -P--C-C--GWQG--C--C----GC-HG-C--PWQC-C---WGG--C-QDLNYC--H-PC

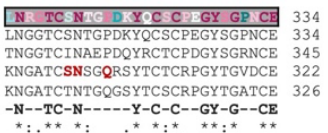

Extended Data Fig 2. Conservation analysis of Site 3 library positions in activating Notch matrix indicating the sequence identity between human Notch ligands. (C) Sequence alignment depicting conservation at each residue position. Residues mutated in the DLL4 mutant library are

542 highlighted in red. 
a

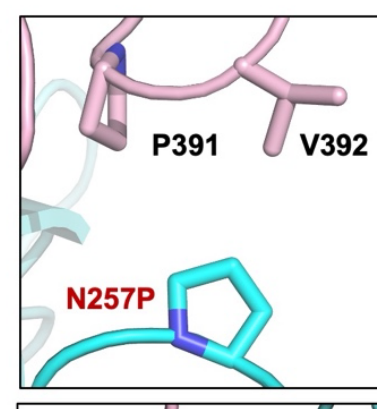

b
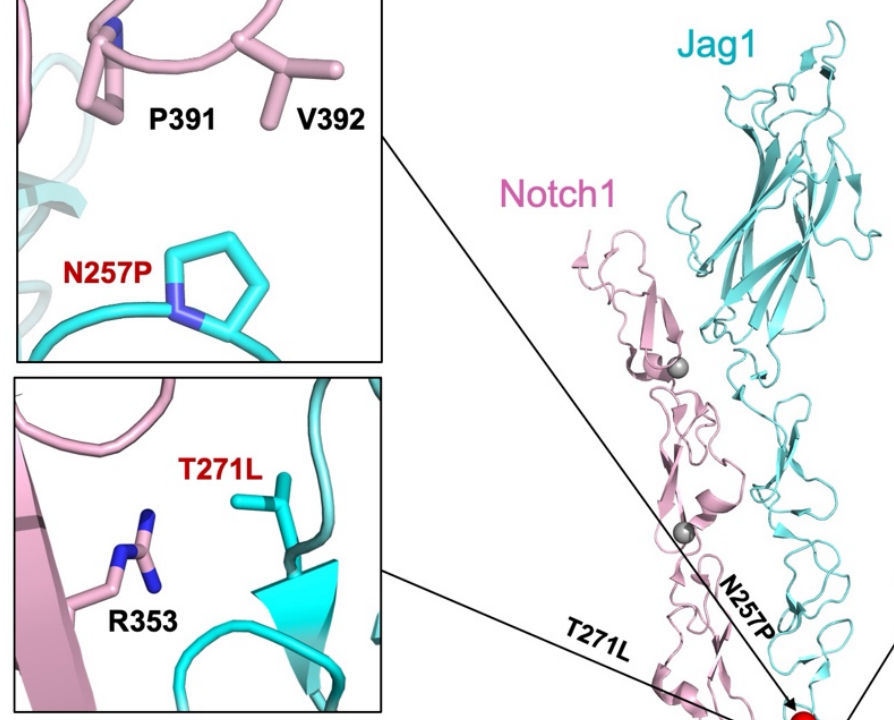

C
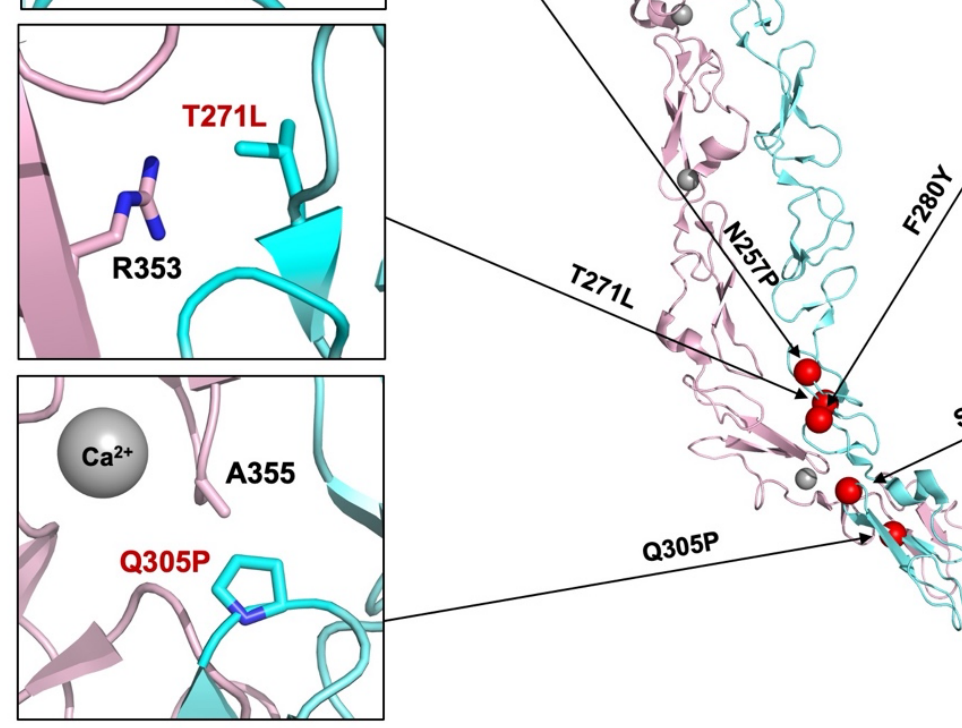

(ent)

Extended Data Fig 3. Mutational analysis of evolved DLL4 Site 3 over Jagged1:Notch1 structure. Cartoon representation showing the structural context of DLL4.v2 mutants in a model of the rat Jag1-Notch1 complex (PDB ID:5UK5). (A), (B), (C), (D), and (E) are zoom panels atomic distances atoms measured in angstroms. 
a

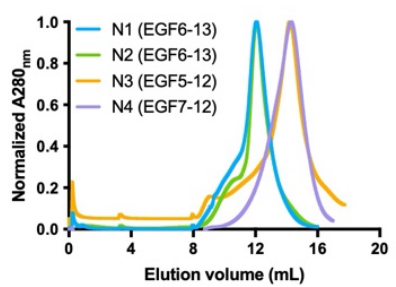

d

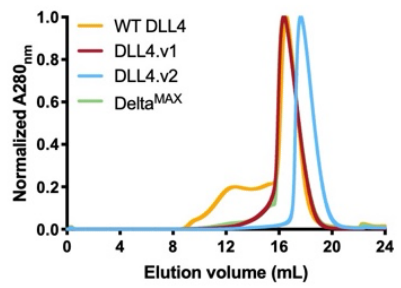

g

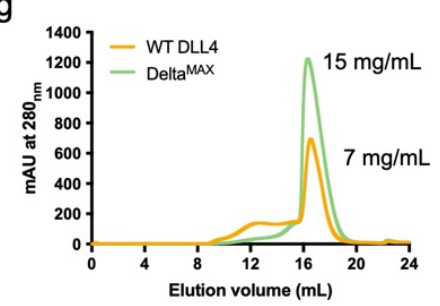

b

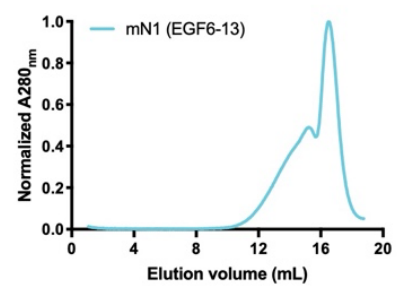

e

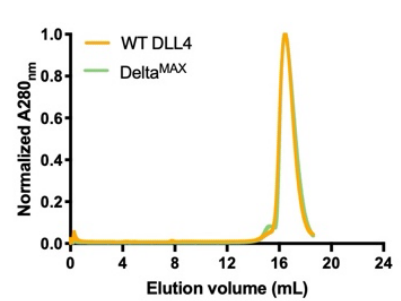

C

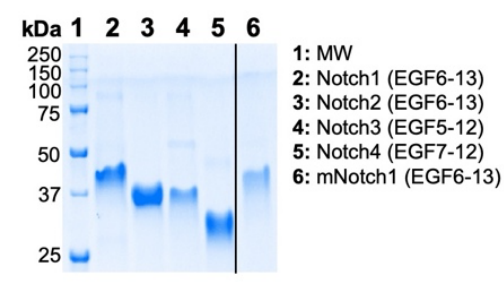

f

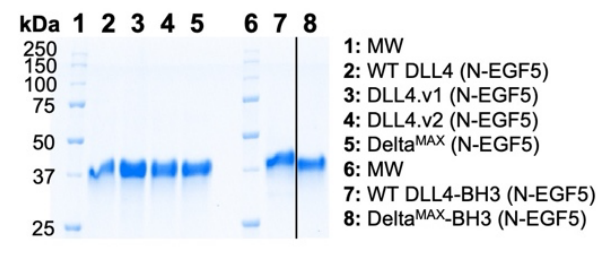

Extended Data Fig 4. Purification of Notch and DLL4 fragments for SPR studies and signaling assays. (A-B) SEC chromatograms from the purification of the ligand-binding regions of human Notch1-4 (A) and murine Notch1 (B). All proteins were purified by Ni-NTA followed by size exclusion chromatography using a Superdex S75 column. (C) SDS-PAGE gels showing the purity and molecular weight of each Notch construct. (D) SEC chromatograms from the purification of DLL4, DLL4.v1, DLL4.v2, and Delta ${ }^{\mathrm{MAX}}$ proteins. All proteins were purified by Ni-NTA followed by size exclusion chromatography using a Superdex S200 column. (E) SEC chromatograms from the purification of C-termini biotinylated WT DLL4 and Delta ${ }^{\mathrm{MAX}}$ proteins. (F) SDS-PAGE gels showing the purity and molecular weight of each DLL4 construct. (G) SEC chromatograms from 1L protein preps were overlaid to highlight the increased yield of recombinant Delta ${ }^{\mathrm{MAX}}$ compared to WT DLL4. 
Extended Data Fig 5. SPR binding assays for mNotch1. SPR binding isotherms were fitted to 


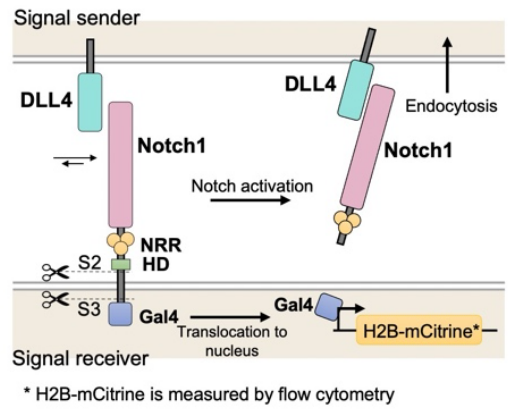

b

Overview of U2OS Notch1/2/3-Gal4 reporter cell lines
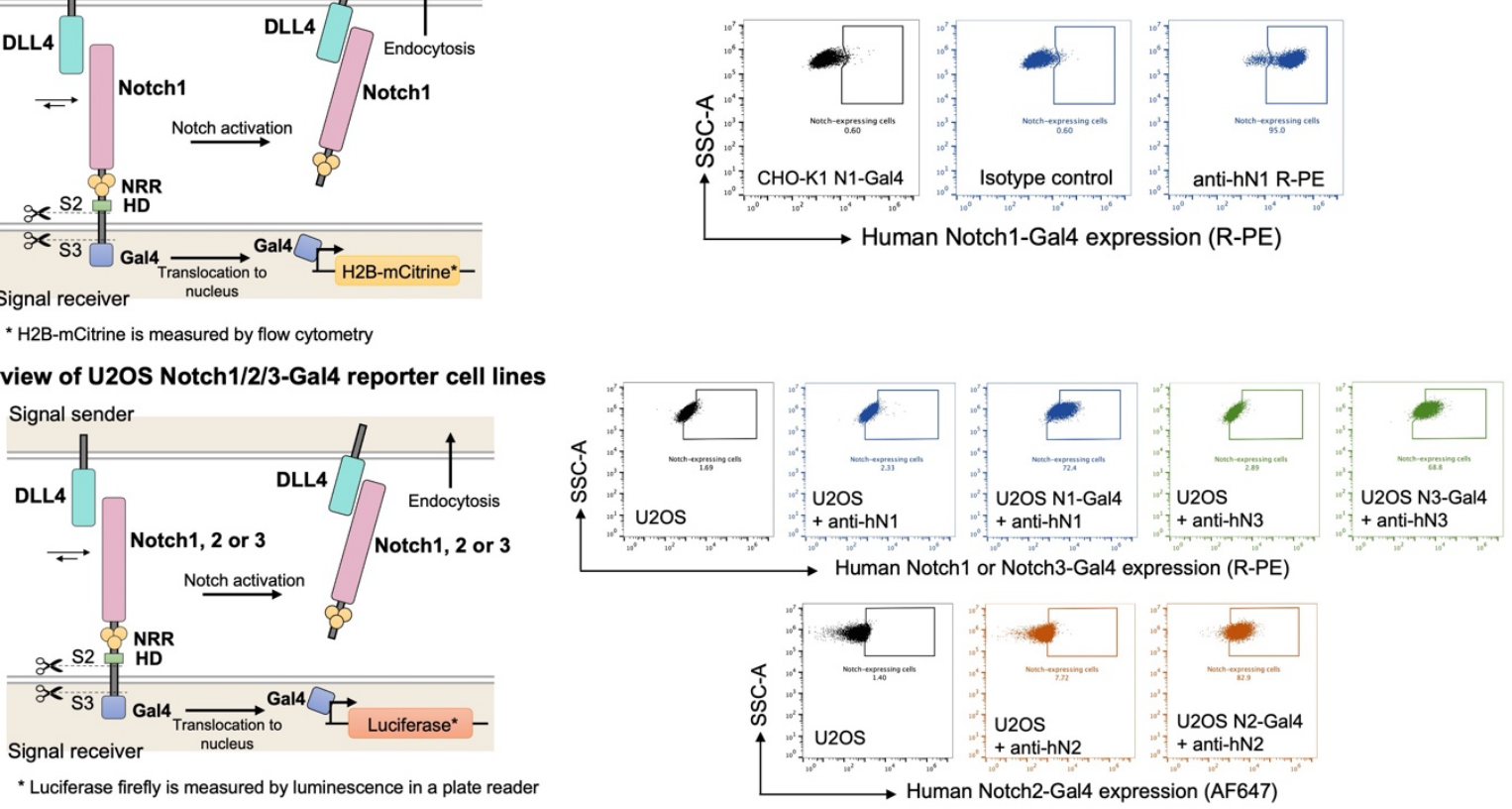

615 Extended Data Fig 6. Notch reporter cell lines used in this study. Cartoon schematics

616 describing the fluorescent (A) and luminescent (B) Notch-Gal4 reporter systems used for signaling 617 assays. Flow cytometry dot plots depict the staining of each cell line with Notch-specific antibodies 618 to detect surface expression. 
a
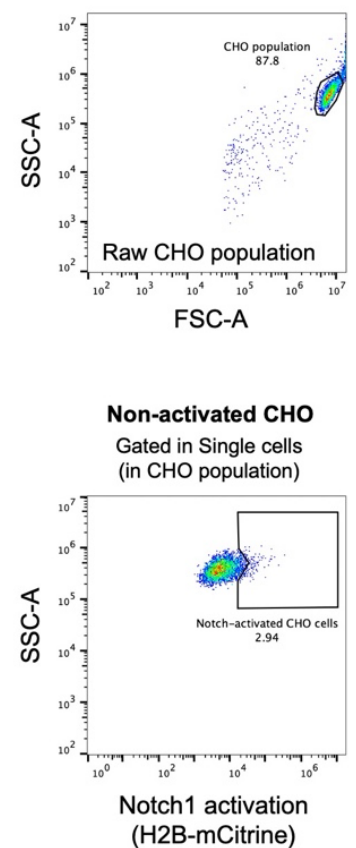
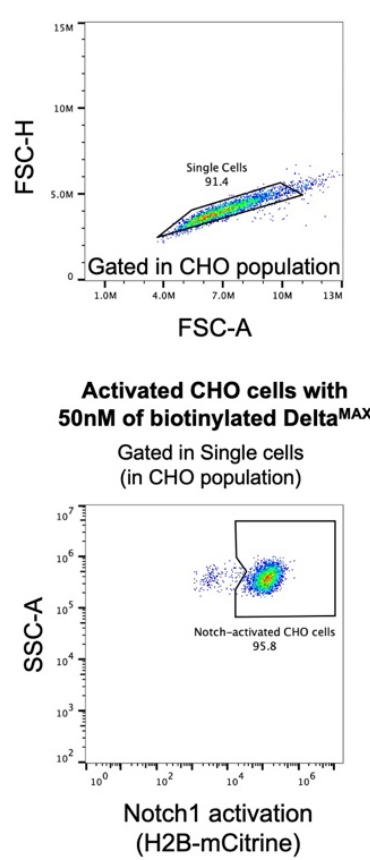

b
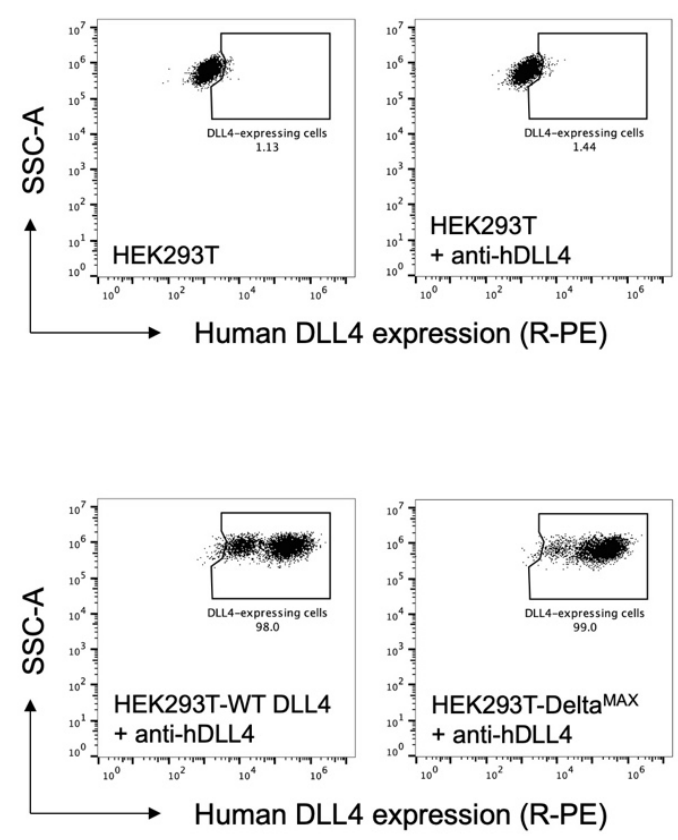

Extended Data Fig 7. Gating strategy for Notch activation assays using CHO-K1 and 635 detection of DLL4 expression in HEK293T stable cell lines. (A) DLL4 variants were nonspecifically adsorbed to 96-well tissue culture plates or immobilized to streptavidin plates. Next, CHO-K1 Notch1-Gal4 cells were added to plates and Notch activation was measured by flow cytometry. The gating strategy to quantify Notch1 activation based on expression of H2B-mCitrine using CHO reporter cells is detailed. HEK293T were transduced to generate stable cell lines expressing WT DLL4 or Delta ${ }^{\mathrm{MAX}}$ and sorted to normalize the expression of DLL4. (B) Expression of WT DLL4 and Delta ${ }^{\text {MAX }}$ on HEK293T was analyzed with anti-hDLL4 PE antibody using flow cytometry. The sorted cell lines were used for Notch activation assays in co-culture with Notch1,

643 Notch2, and Notch3-U2OS luciferase reporters. 
a

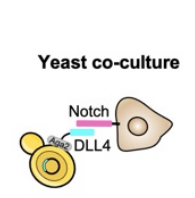

HEK293T co-culture

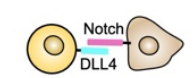

DLL4-coated beads

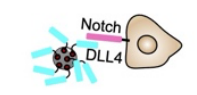

Non-specific adsorption

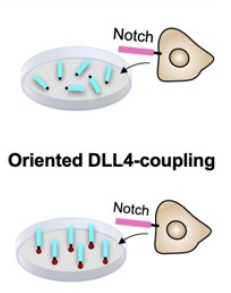

b

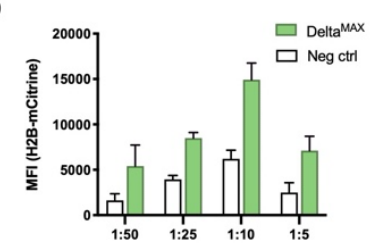

C

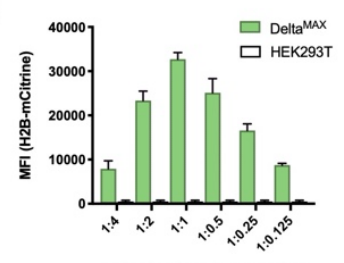

d

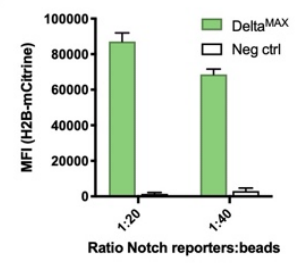

e
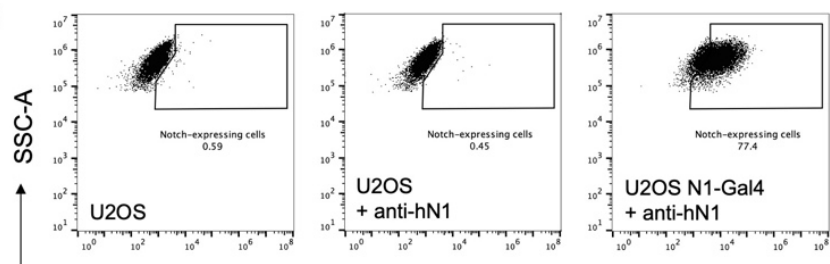

$\rightarrow$ Human Notch1-Gal4 expression (R-PE)
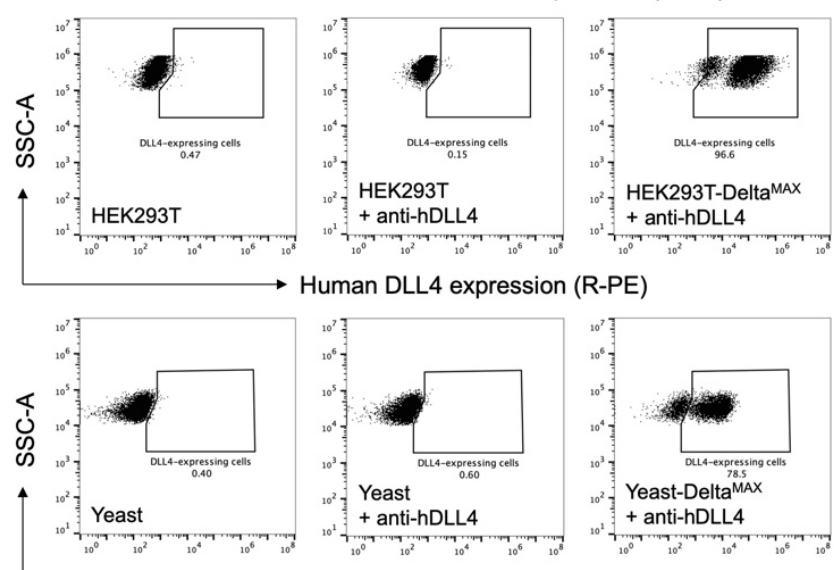

Human DLL4 expression (R-PE)
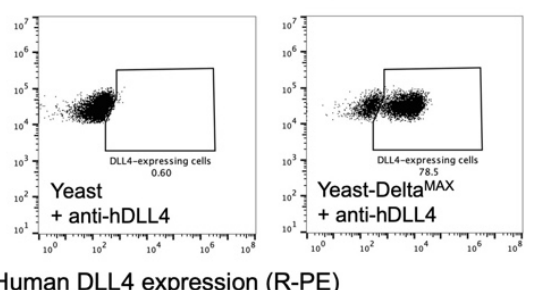

Extended Data Fig 8. Optimization for all-in-one assay using Delta ${ }^{\text {MAX }}$. (A) Cartoon were co-cultured with $\mathrm{CHO}-\mathrm{K} 1$ reporter cells for $24 \mathrm{~h}$ at different ratios and fluorescence was measured by flow cytometry. (C) HEK293T cells expressing Delta ${ }^{\mathrm{MAX}}$ were co-cultured with Notch1 reporter cells at various ratios for $24 \mathrm{~h}$ and Notch activation was measured by flow cytometry. (D) Magnetic beads were pre-coated with biotinylated Delta ${ }^{\mathrm{MAX}}$ and co-cultured with CHO K1 reporter cells at ratios of 1:20 or 1:40 to stimulate Notch activation. (E) Flow cytometry dot plots depict the expression level of Notch1 and Delta ${ }^{\mathrm{MAX}}$. 
a

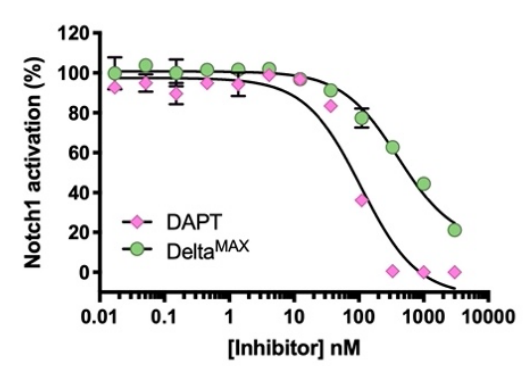

b
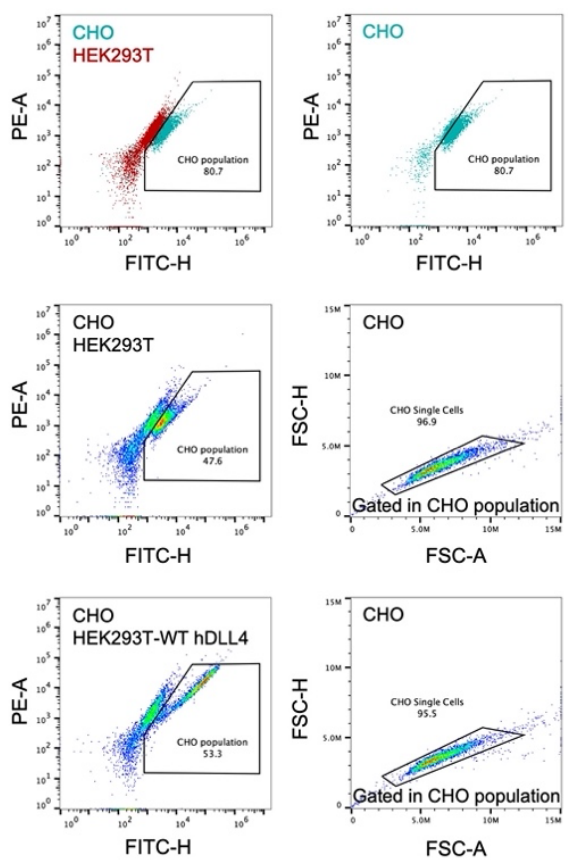
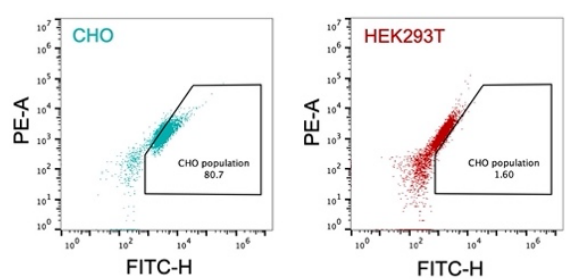

FITC-H
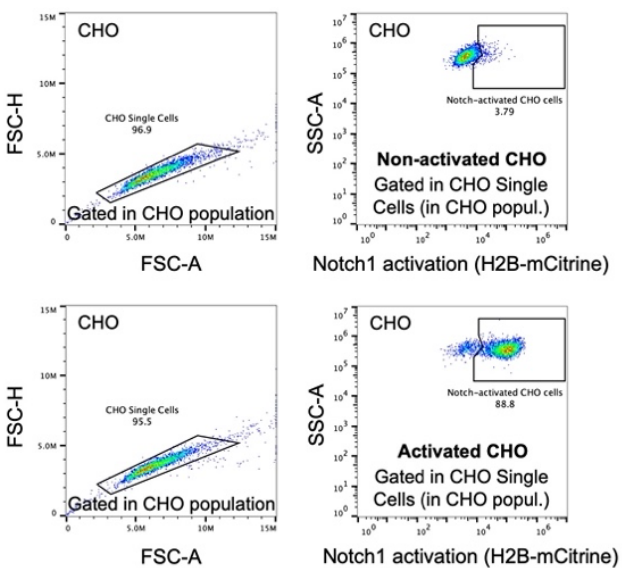

Extended Data Fig 9. Co-culture inhibition using CHO-K1 cells, DAPT, or Delta ${ }^{\mathrm{MAX}}$ as soluble antagonists. (A) Dose-titration assay comparing the inhibition potency of DAPT and Delta $^{\mathrm{MAX}}$. Fluorescent Notch1 reporter cells were cultured in a 1:1 ratio with HEK293T cells stably expressing WT DLL4. (B) Summary of the gating strategy used for flow cytometry to differentiate between HEK293T and CHO cell signals. The basal expression of H2B-mCitrine in CHO cells was used as a criterion to distinguish between cell types. Notably, the population identified in the middle and bottom panels (Notch1 reporter CHO cells) corresponds to approximately 50\% of the total cells, which is consistent with a 1:1 ratio of $\mathrm{CHO}$ cells to $293 \mathrm{~T}$ cells. 

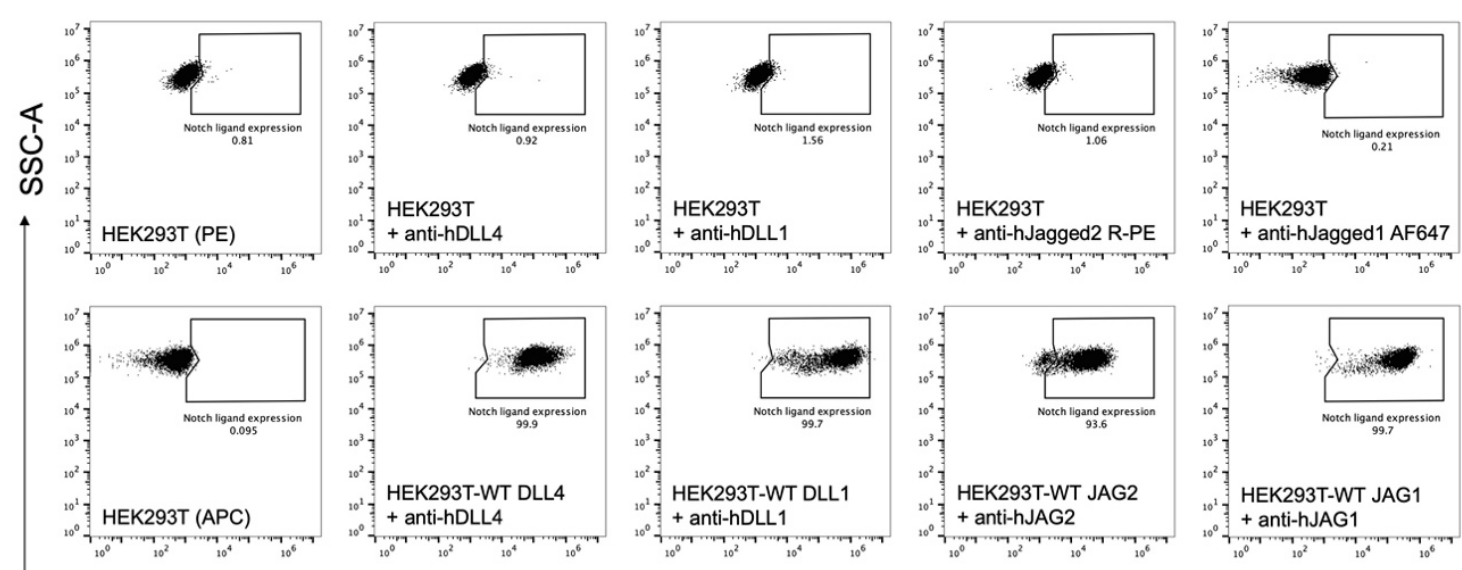

$\rightarrow$ Human DLL1, DLL4, JAG2 (R-PE

PE), or JAG1 (AF647) expression

690 Extended Data Fig 10. Detection of Notch ligands expressed on HEK293T stable cell lines

691 by flow cytometry. HEK293T stable cells lines expressing WT DLL4, DLL1, JAG1, or JAG2

692 were stained with specific antibodies targeting the ECDs of each Notch ligand and measured by

693 flow cytometry. The expression of Notch ligands, as well as sequencing results, validated these

694 stable cell lines. 